Supporting info - part 3 for the paper:

Computational Mutagenesis at the SARS-CoV-2 Spike Protein/Angiotensin-Converting Enzyme 2 Binding Interface: Comparison with Experimental Evidences

Erik Laurini ${ }^{1, \ddagger}$, Domenico Marson ${ }^{1, \ddagger}$, Suzana Aulic ${ }^{1}$, Alice Fermeglia ${ }^{1}$, Sabrina Pricl ${ }^{1,2^{*}}$

${ }^{1}$ Molecular Biology and Nanotechnology Laboratory (MolBNL@UniTS), DEA, University of Trieste, 34127 Trieste, Italy

${ }^{2}$ Department of General Biophysics, Faculty of Biology and Environmental Protection, University of Lodz, 90-136 Lodz, Poland 
Q24D

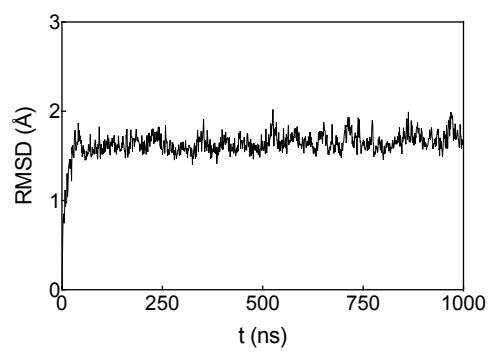

Q24S

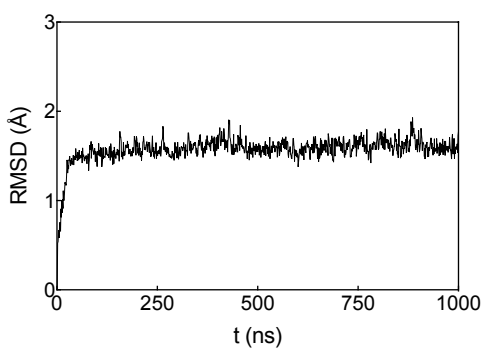

T27D

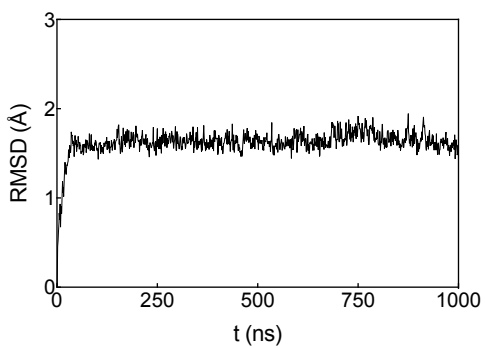

T27S

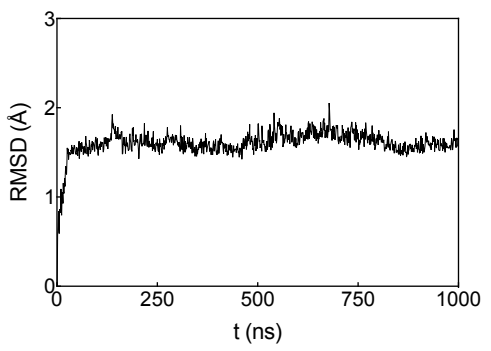

Q24K

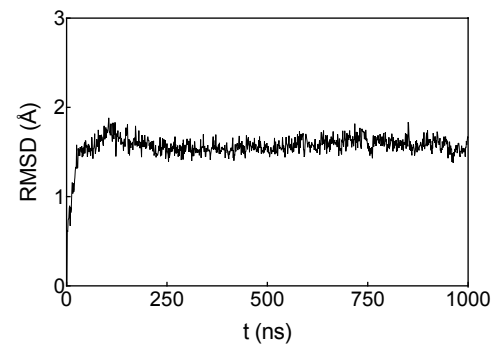

Q24T

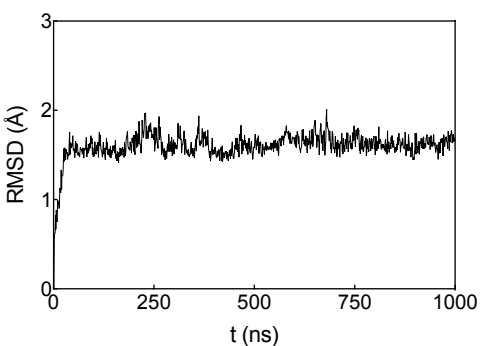

T27K

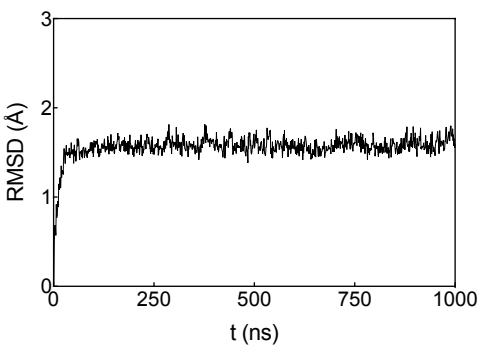

T27W

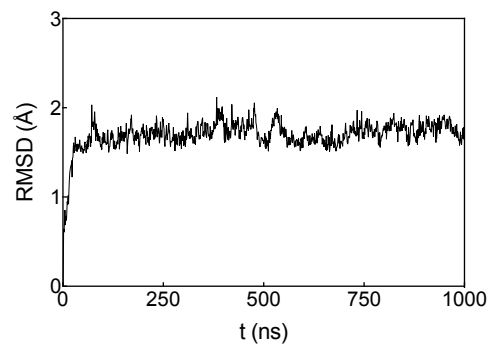


D30E

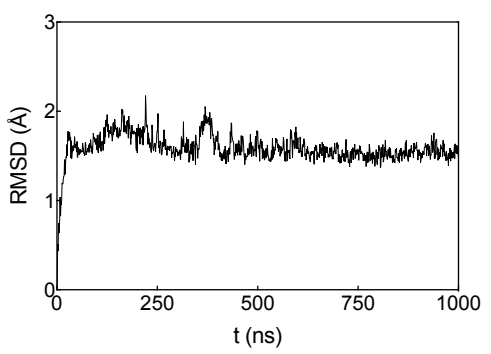

D30S

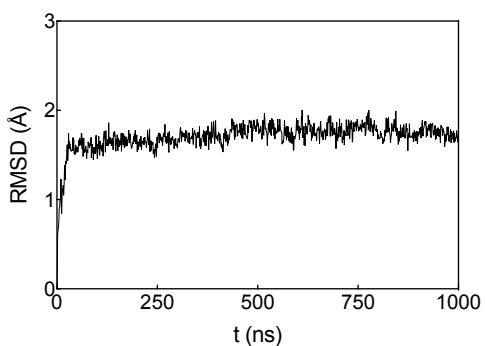

H34D

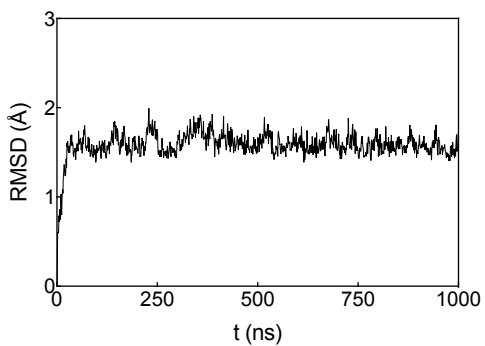

H34S

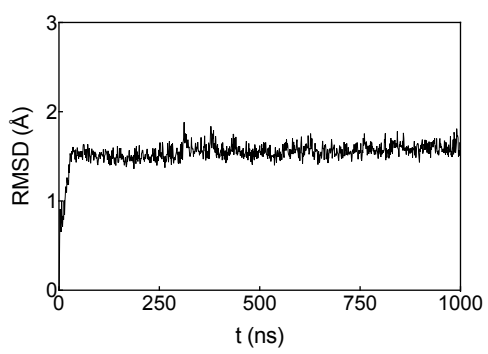

D30K

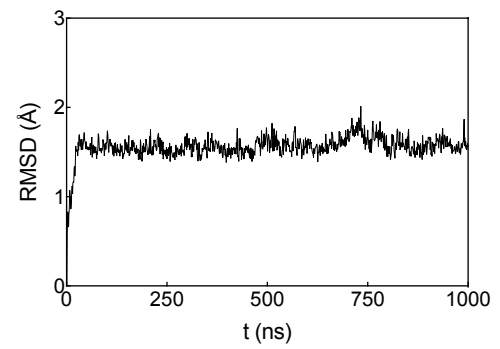

D30T

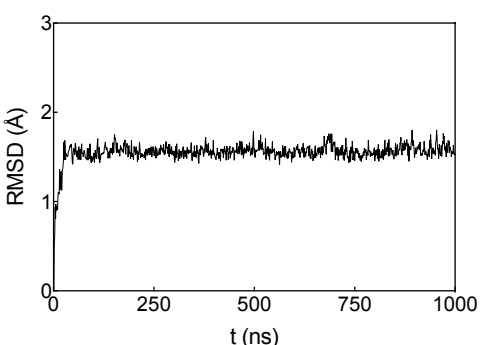

H34K

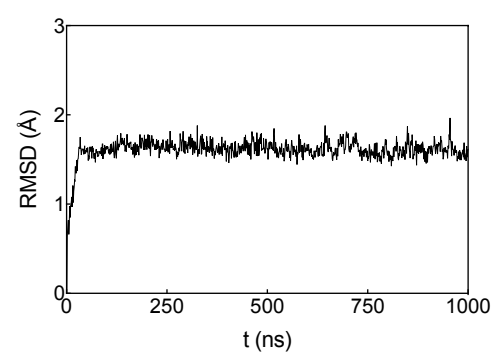

H34T

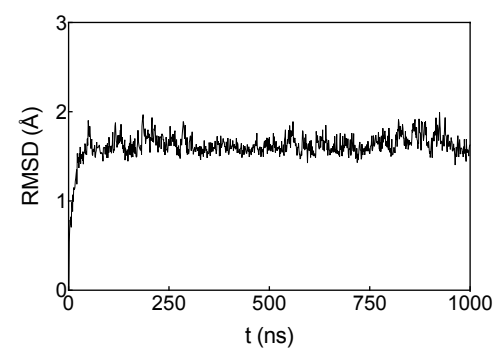

D30I

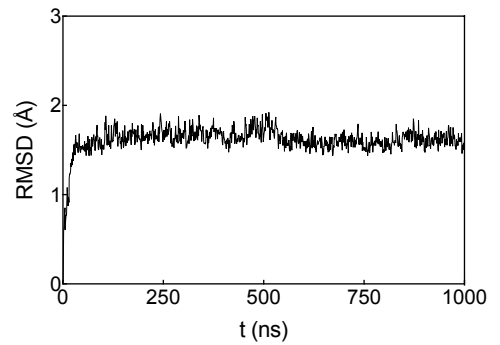

D30W

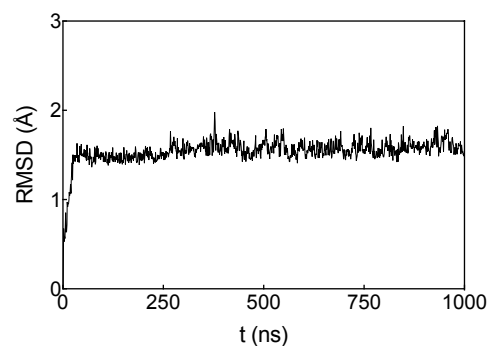

H34I

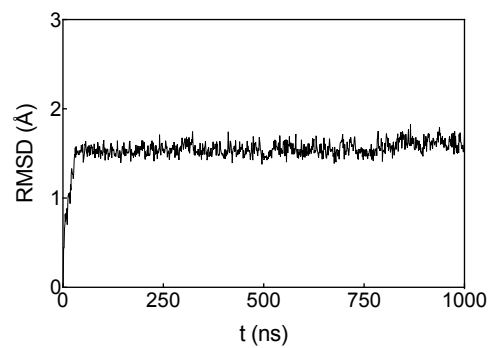

H34W

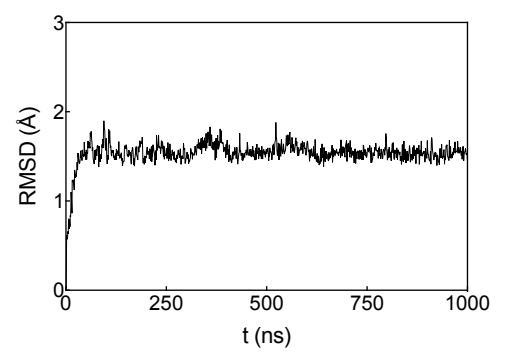


Y83D

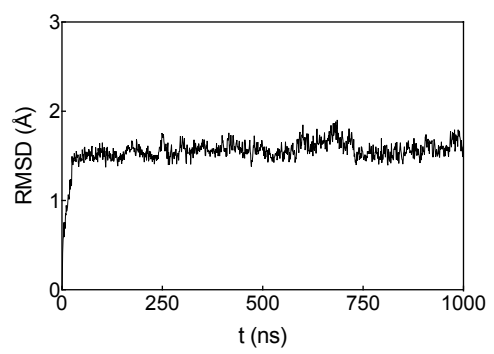

Y83S

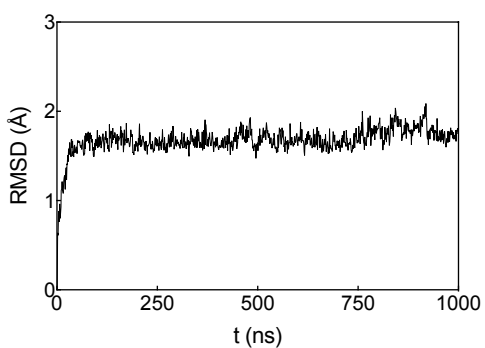

M82D

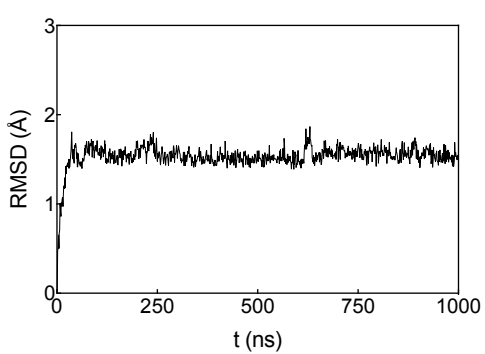

M82S

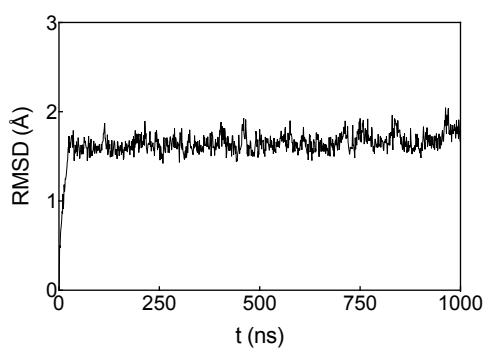

Y83K

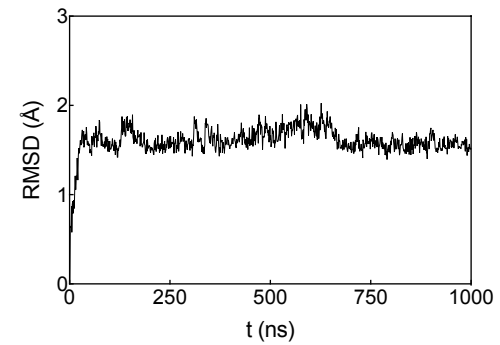

Y83T

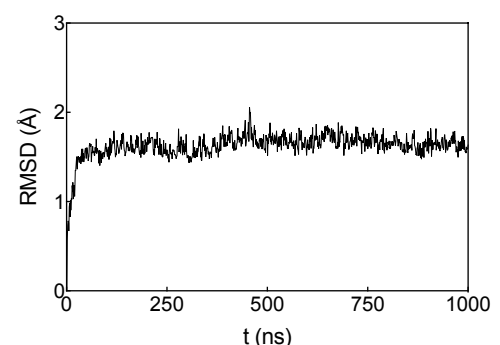

M82K

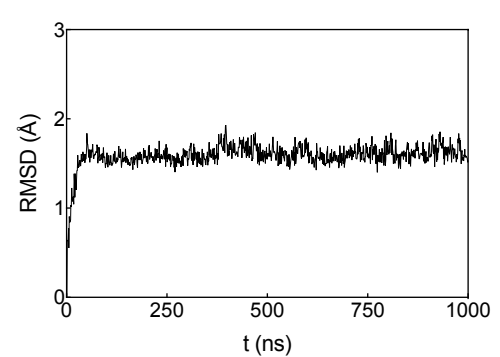

M82T

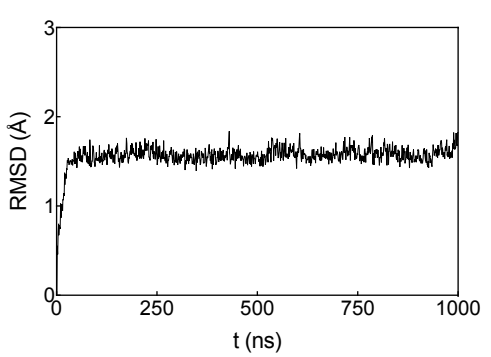

Y83I

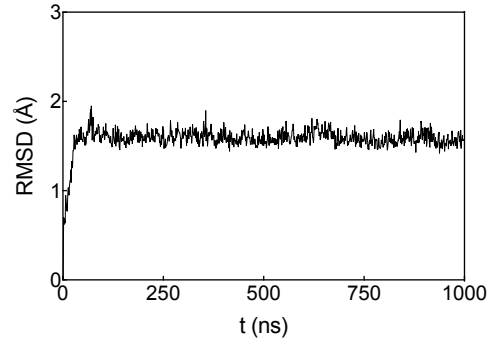

Y83W

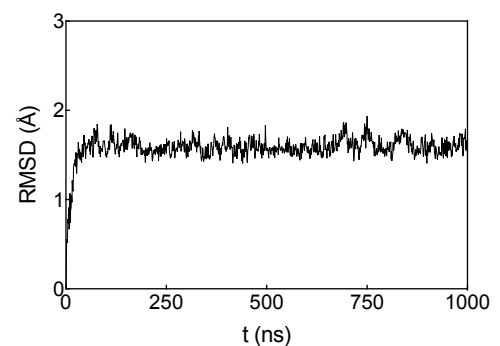

M82

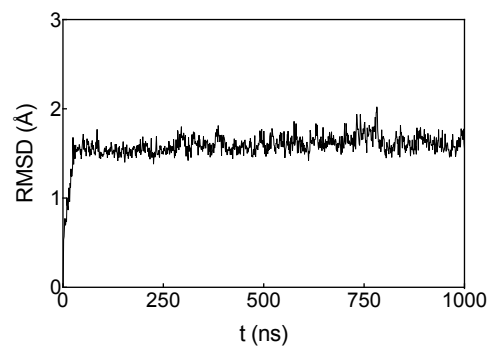

M82W

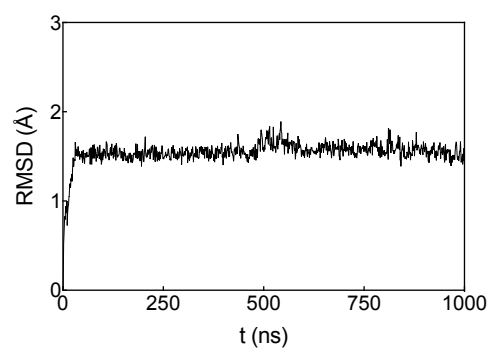


L79D

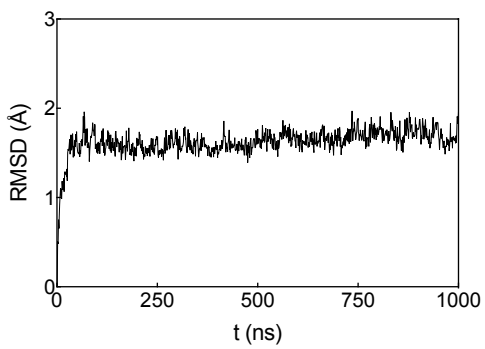

L79S

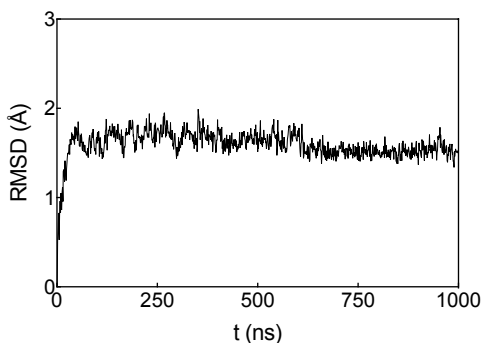

F28D

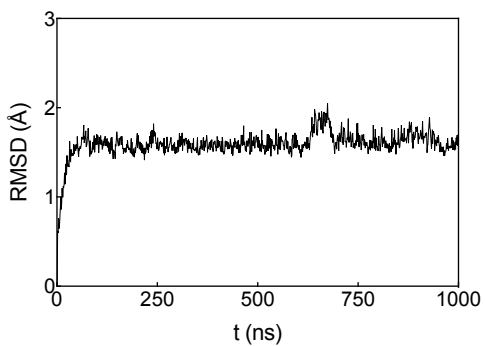

F28S

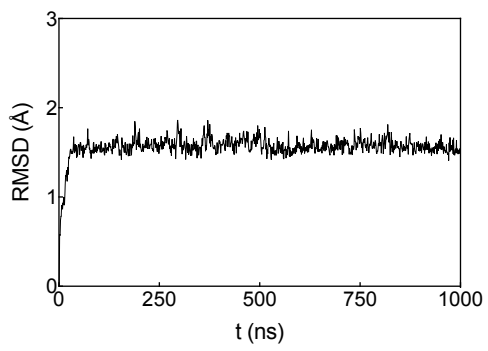

L79K

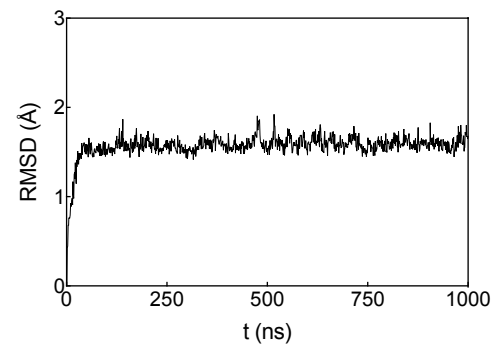

L79T

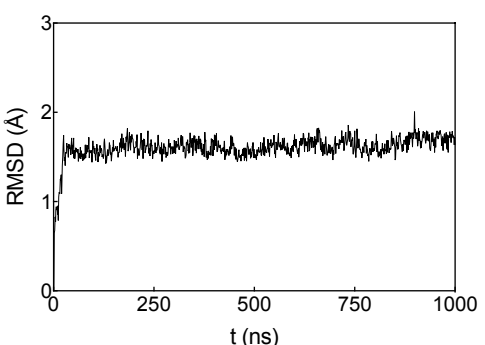

F28K

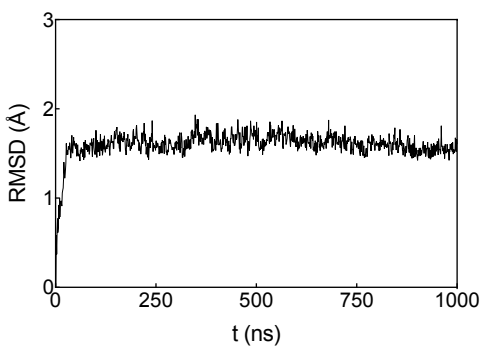

F28T

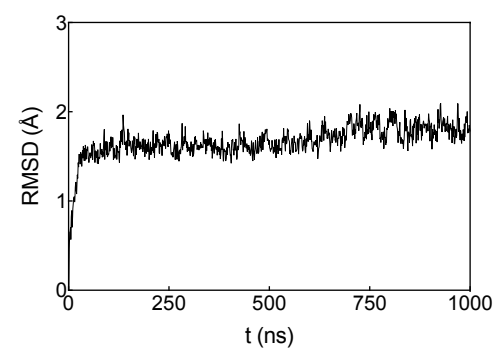

L79I

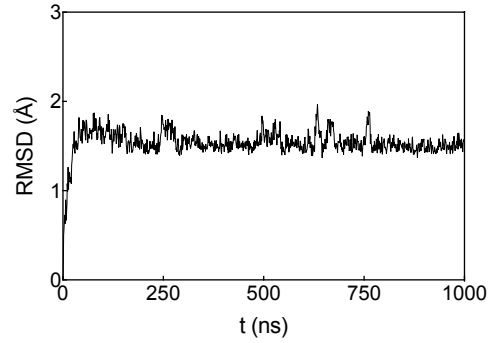

L79W

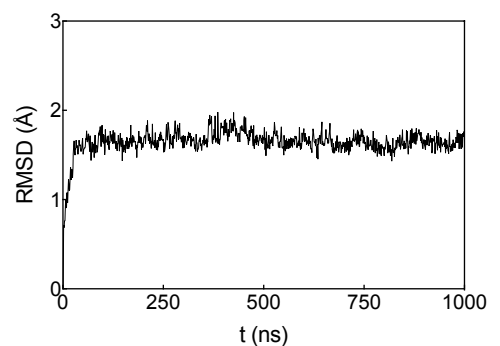

F28I

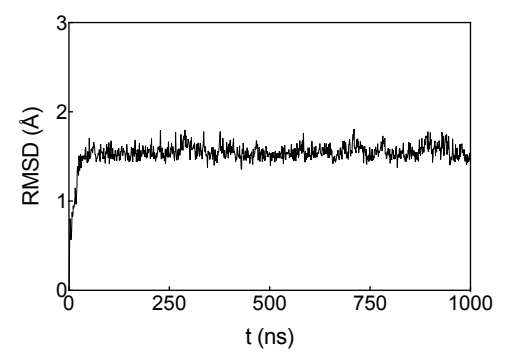

F28W

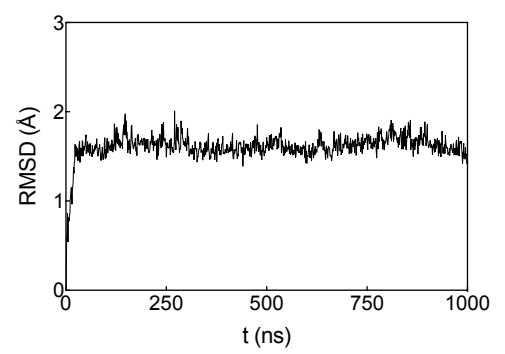


K31D

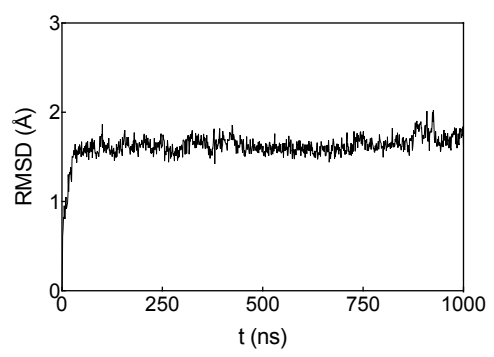

K31S

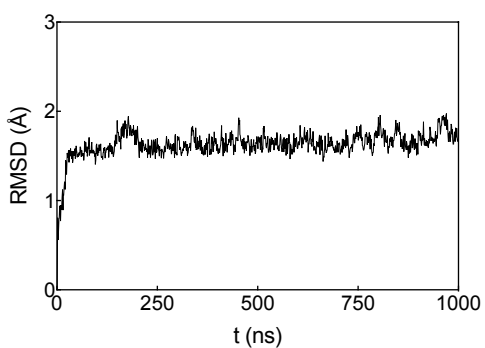

E35D

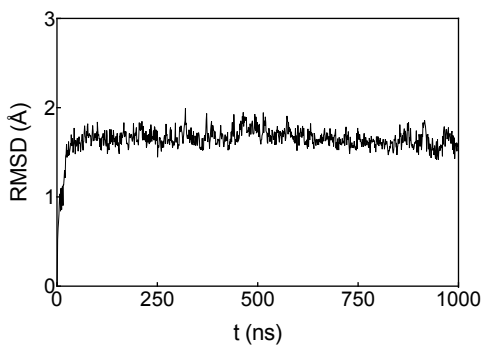

E35S

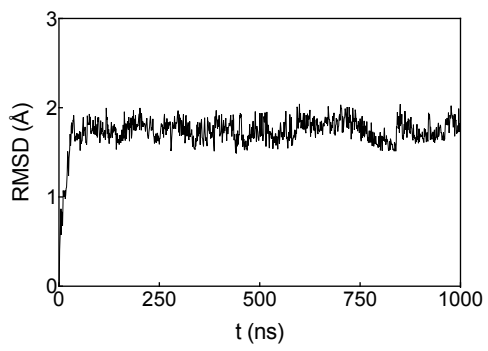

K31R

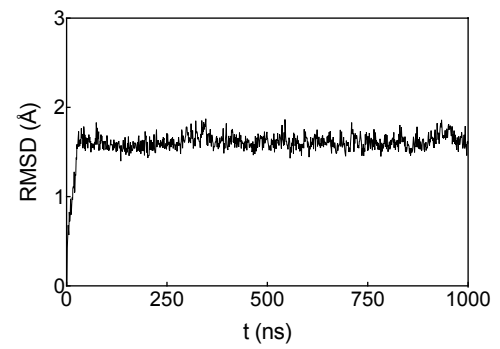

K31T

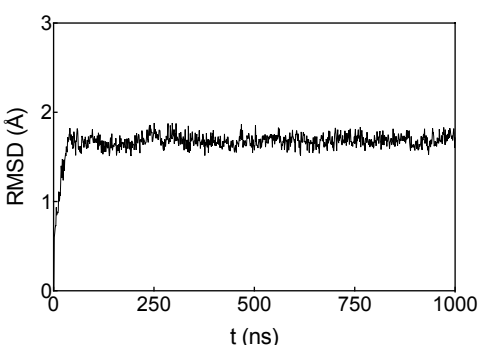

E35K

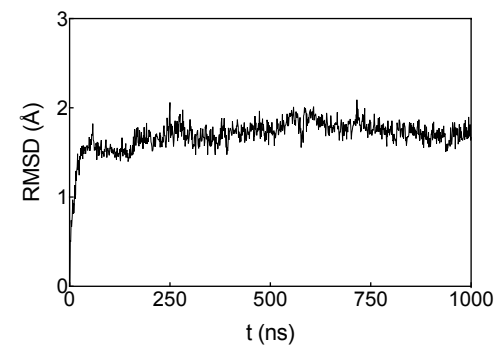

E35T

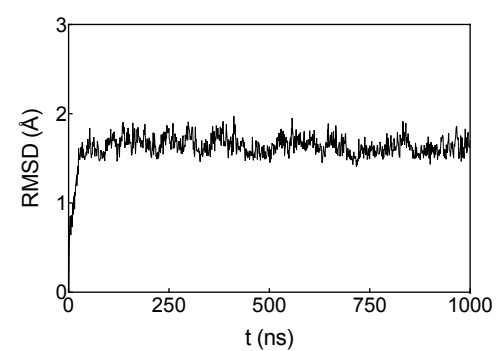

K31I

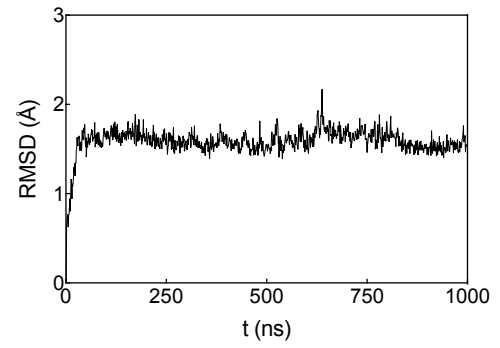

K31W

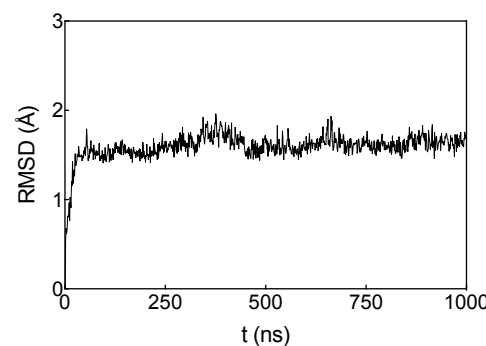

E35I

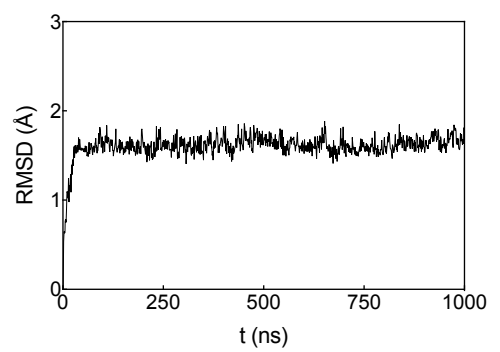

E35W

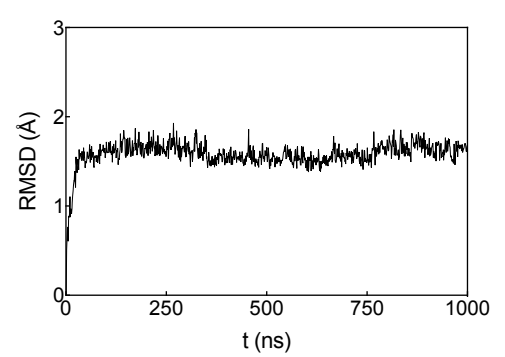


K353D

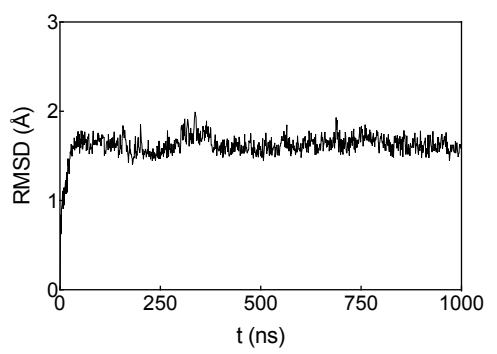

K353S

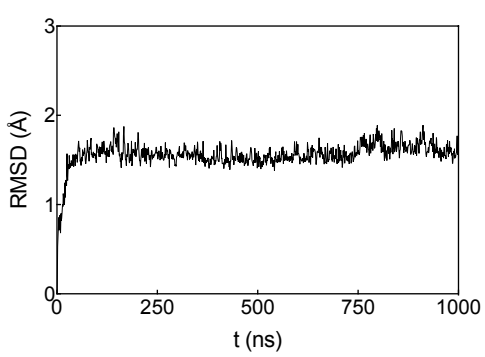

D38E

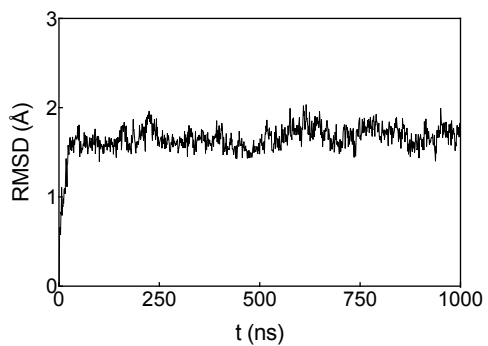

D38S

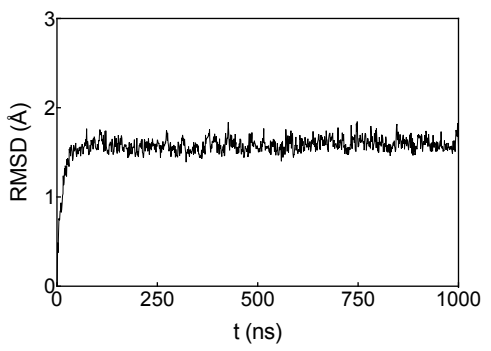

K353R

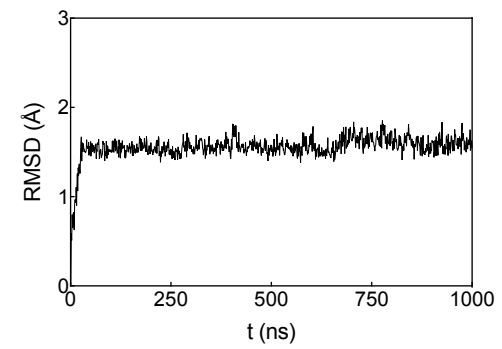

K353T

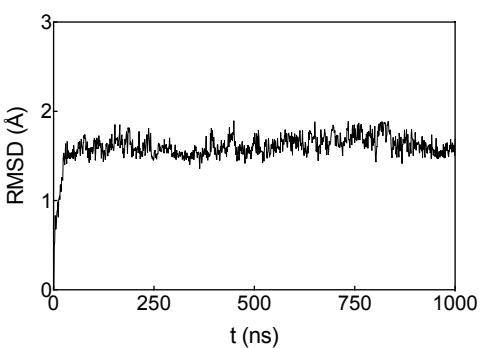

D38K

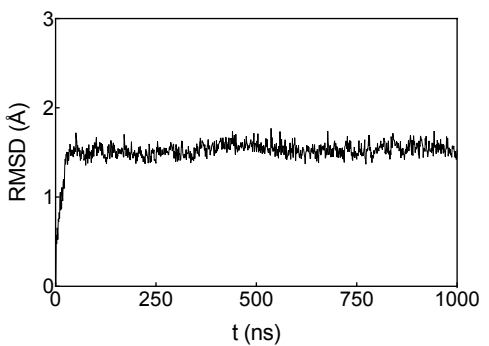

D38T

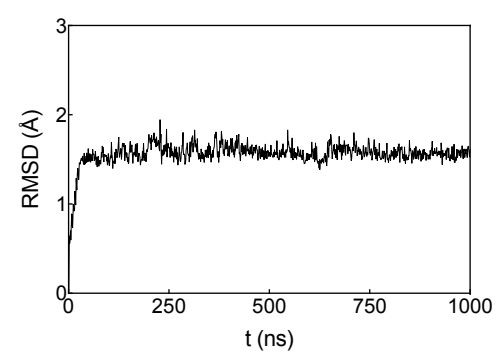

K353I

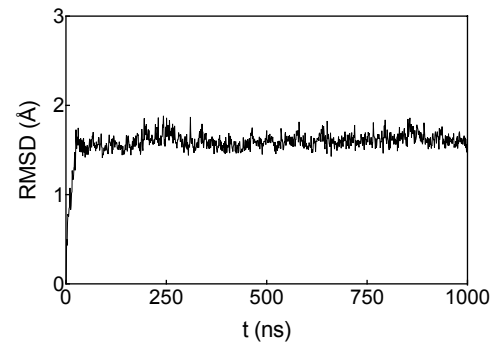

K353W

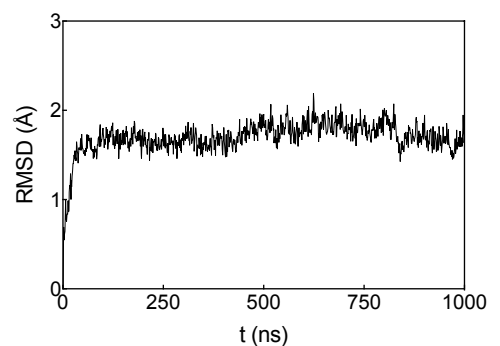

D38I

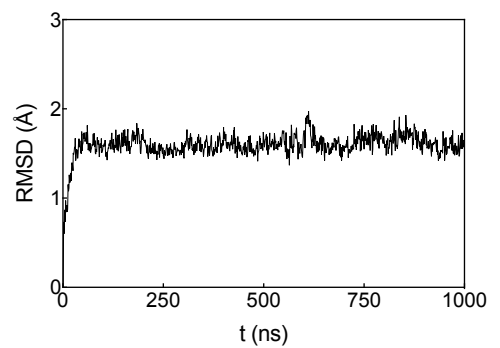

D38W

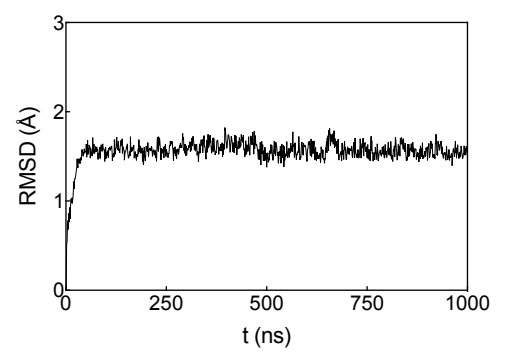


Q42D

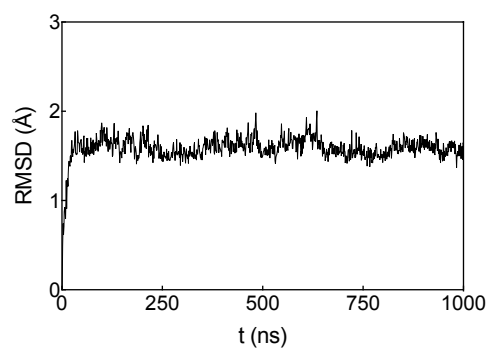

Q42S

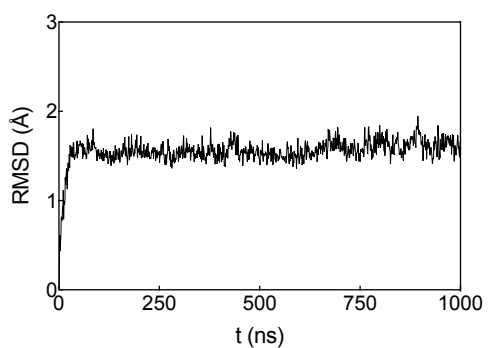

Y41D

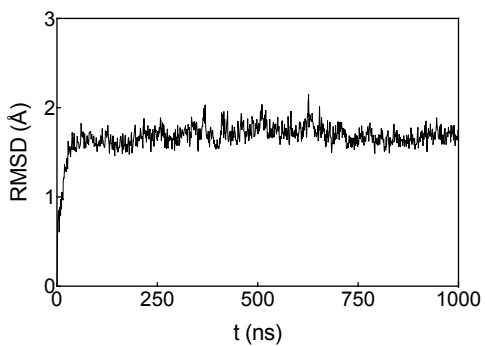

Y41S

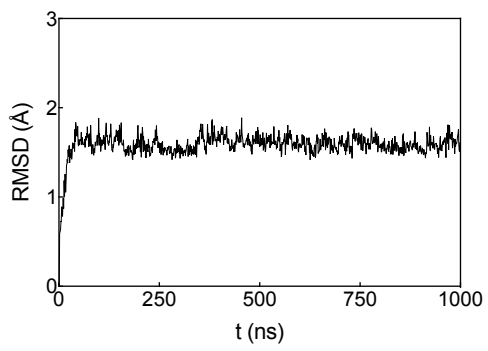

Q42K

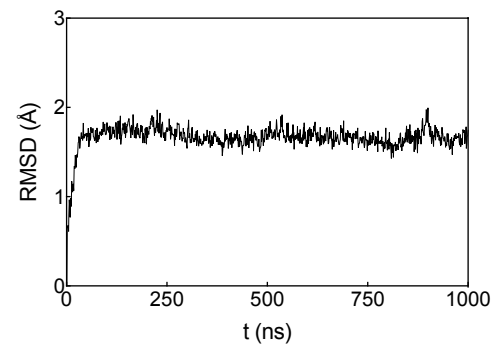

Q42T

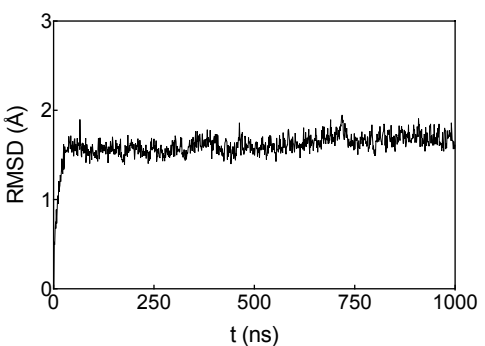

Y41K

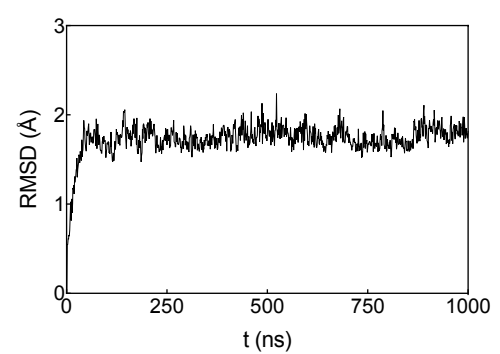

Y41T

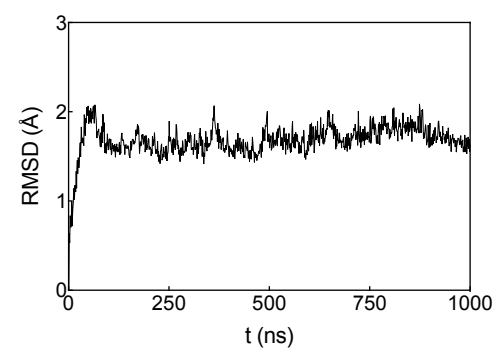

Q42I

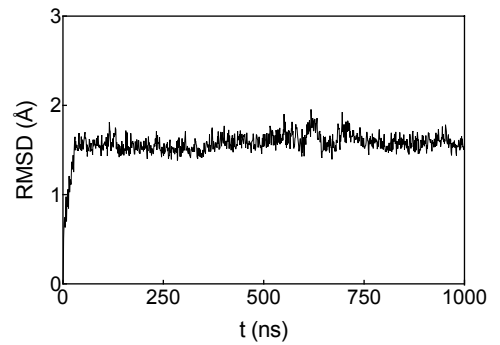

Q42W

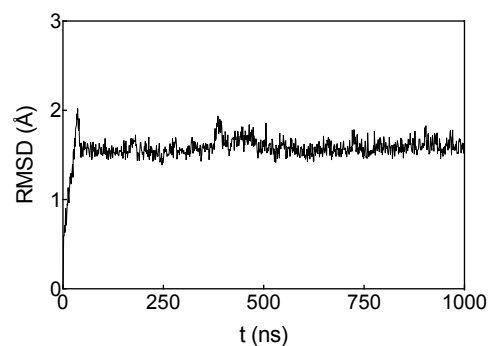

Y41I

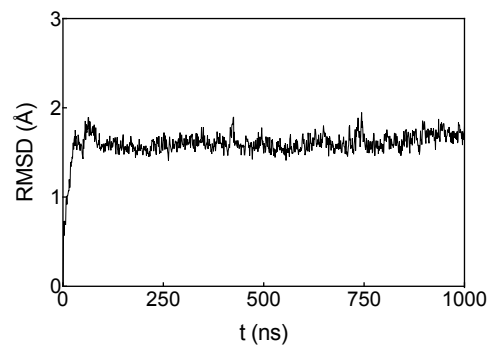

Y41W

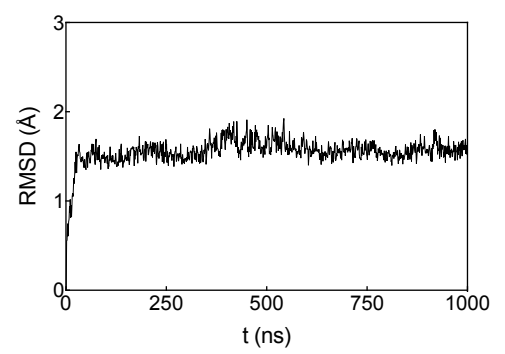


D355E

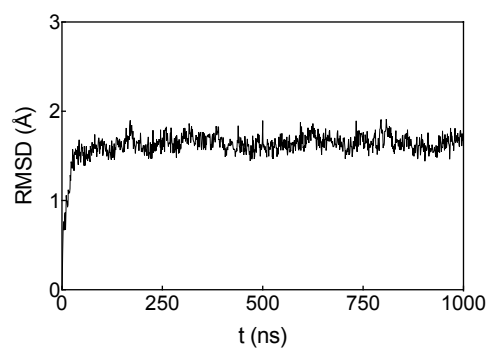

D355S

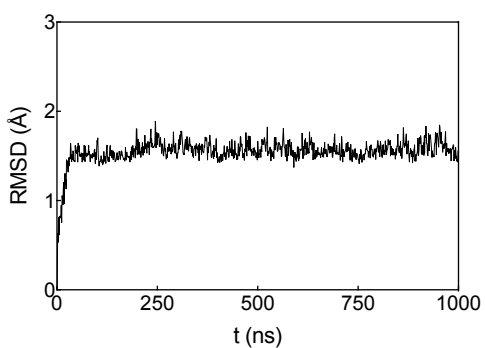

R357D

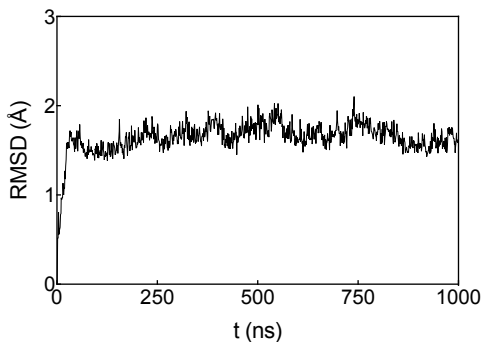

R357S

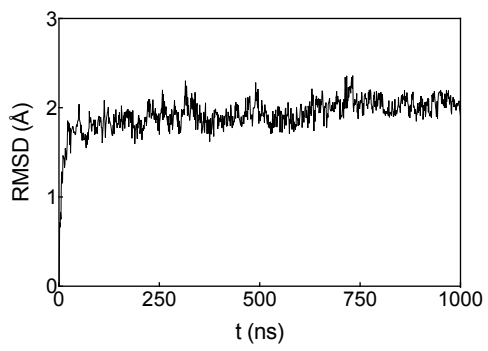

D355K

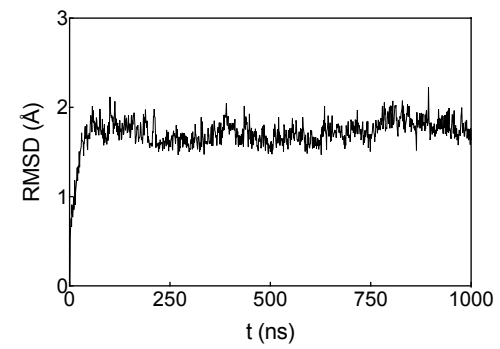

D355T

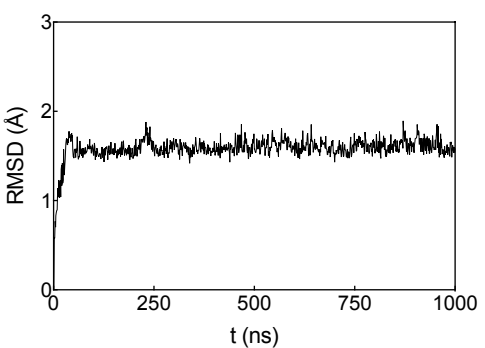

R357K

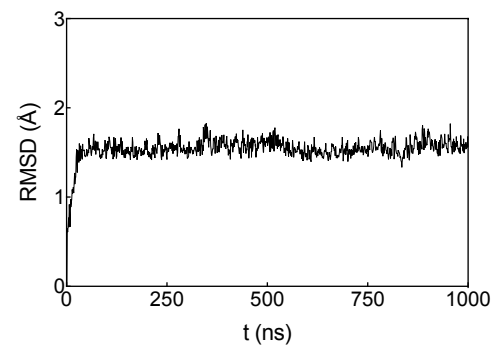

R357T

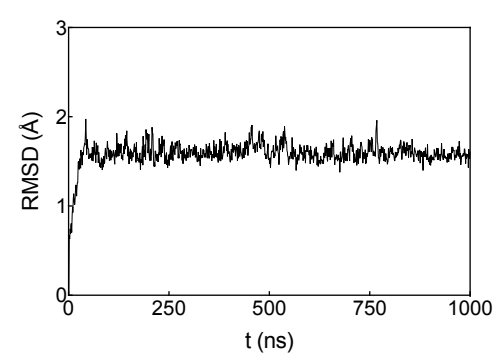

D355I

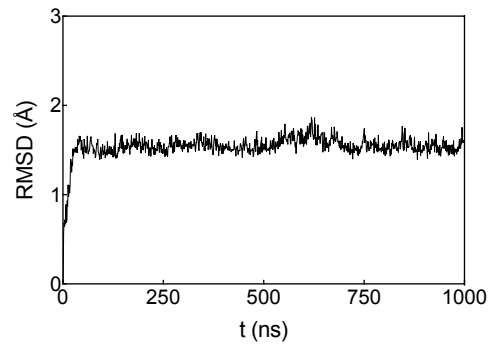

D355W

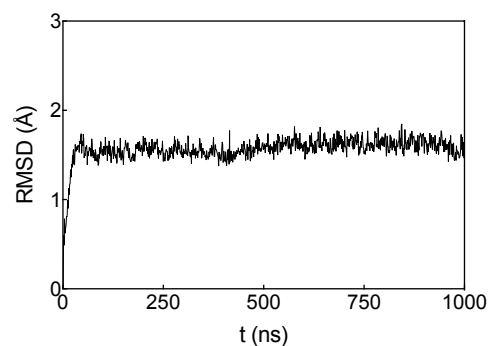

R357I

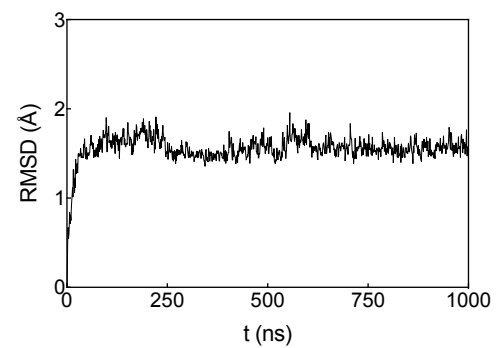

R357W

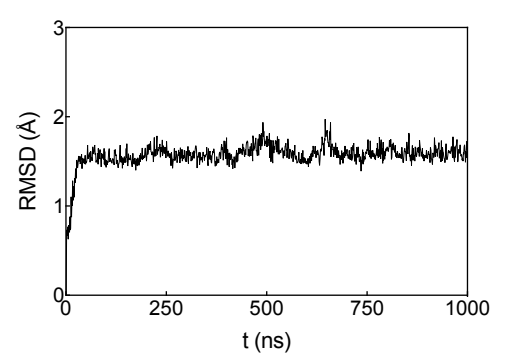


E37D

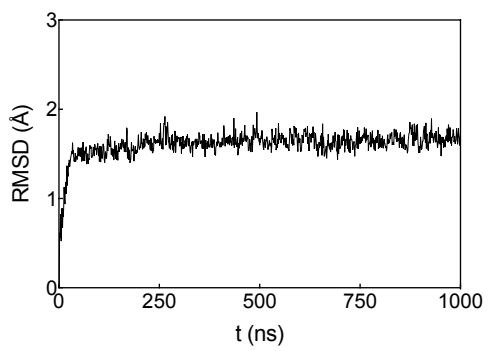

E37S

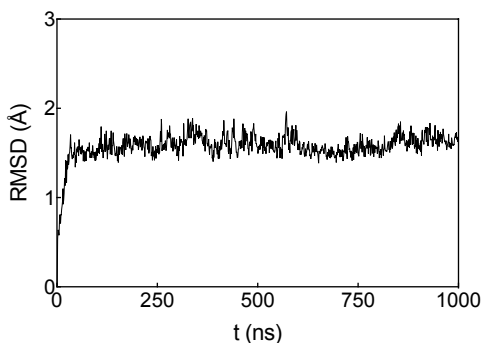

R393D

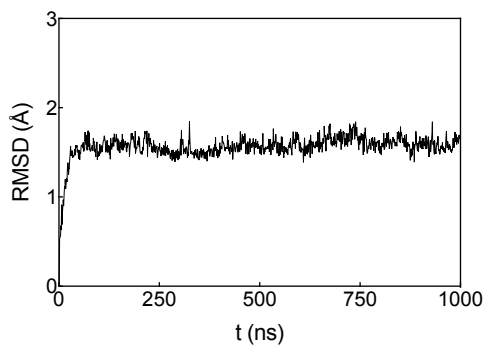

R393S

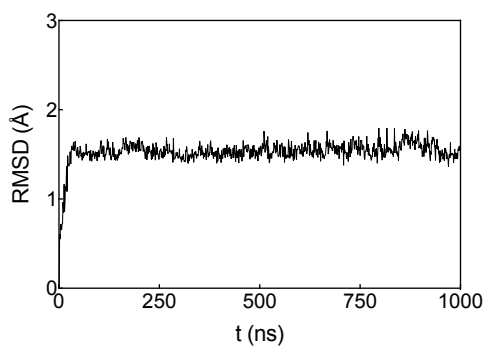

E37K

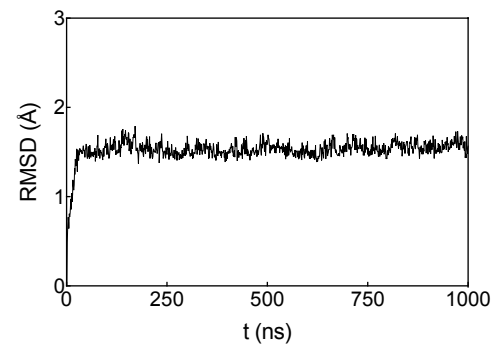

E37T

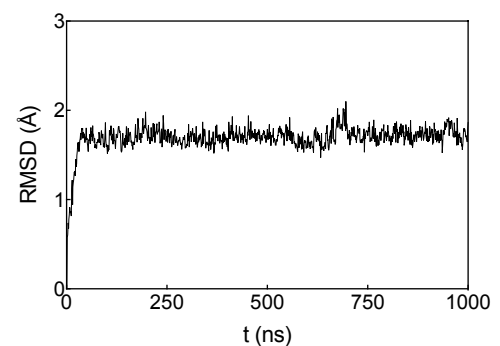

R393K

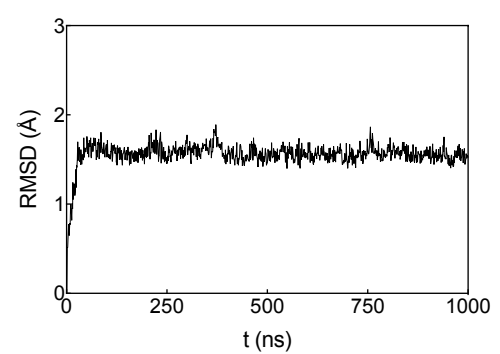

R393T

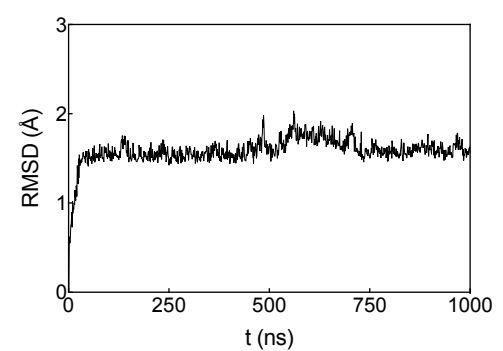

E37I

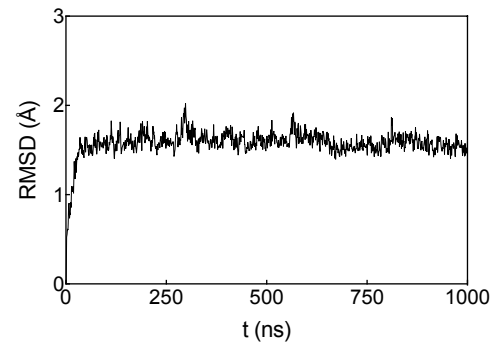

E37W

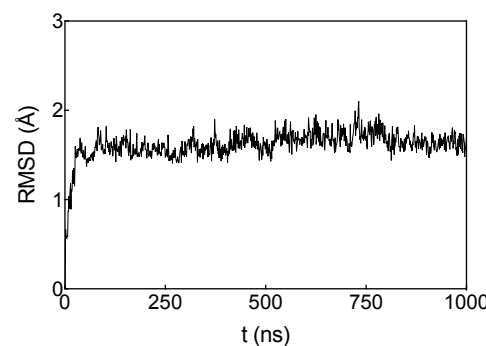

R393I

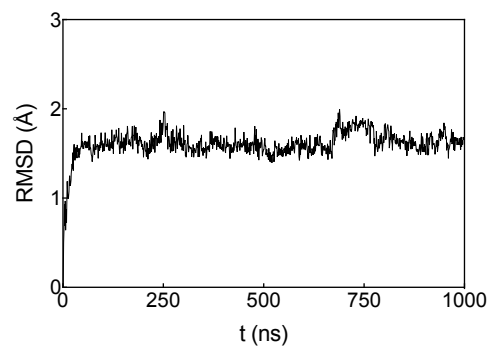

R393W

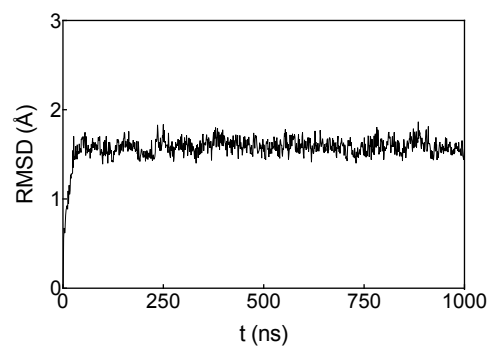

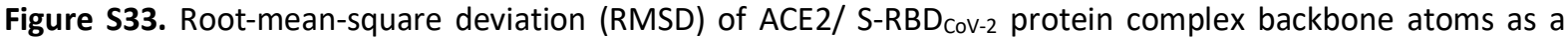
function of MD simulation time for all ACE2 mutants. 
Y449D

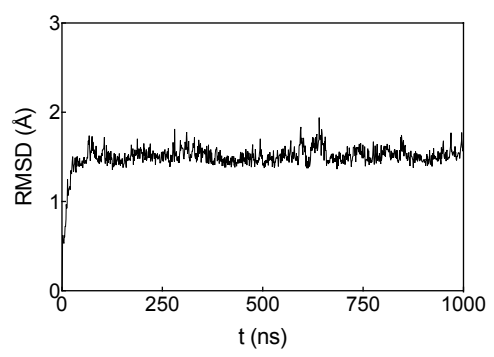

Y449S

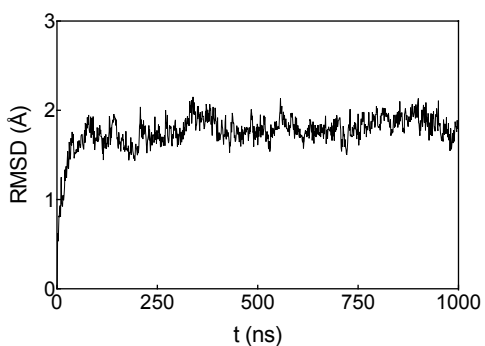

Y453D

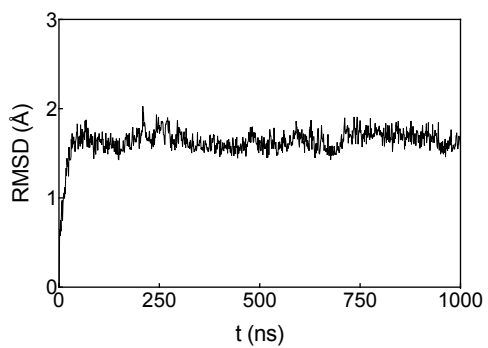

Y453S

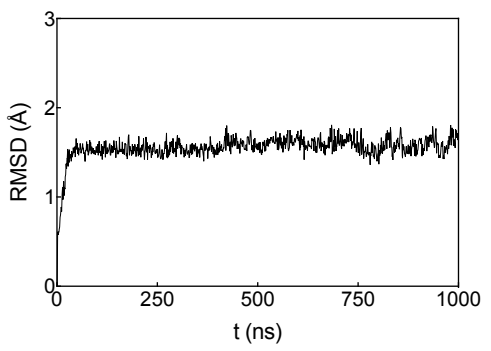

Y449K

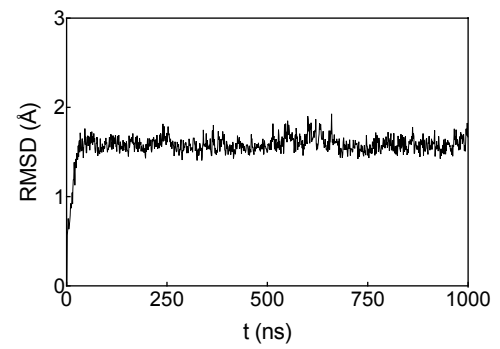

Y449T

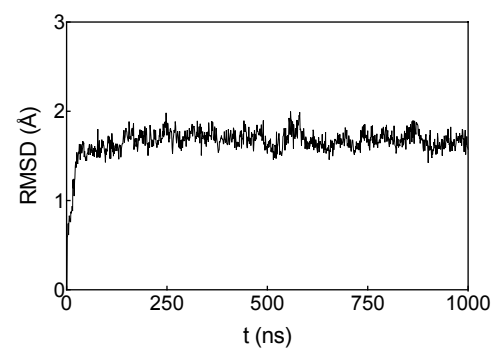

Y453K

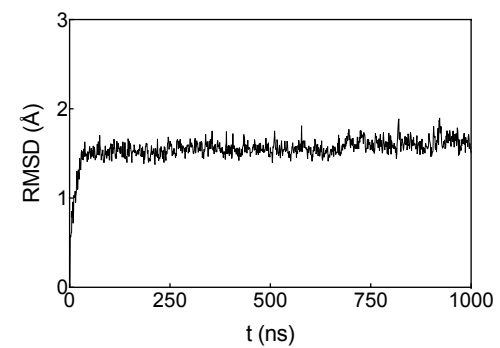

Y453T

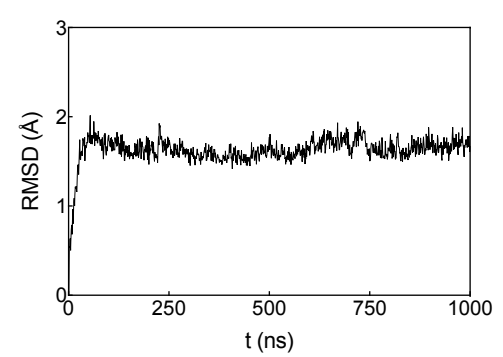

Y449I

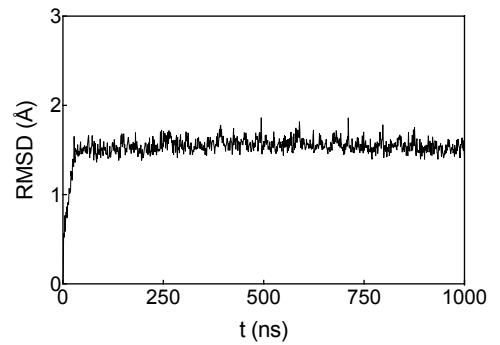

Y449W

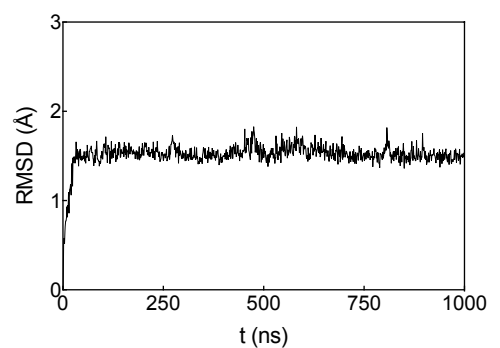

Y453I

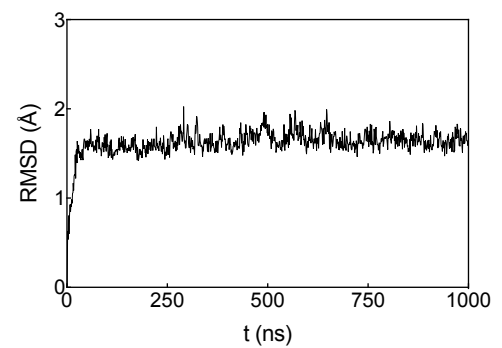

Y453W

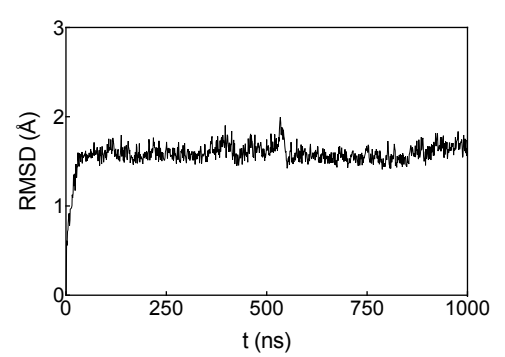


T500D

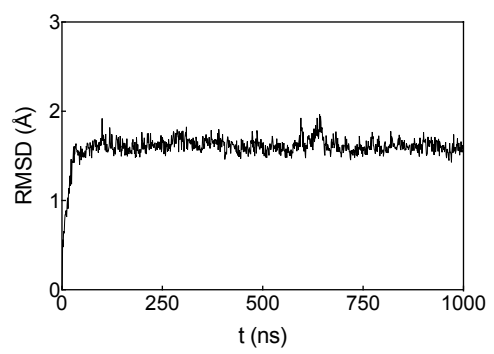

T500S

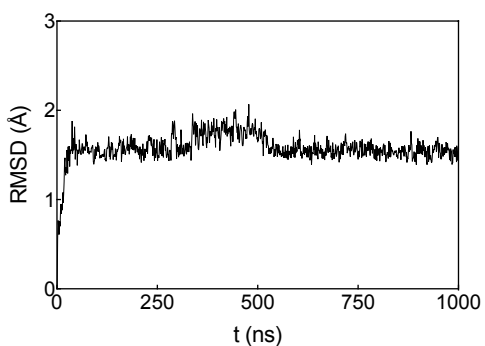

N487D

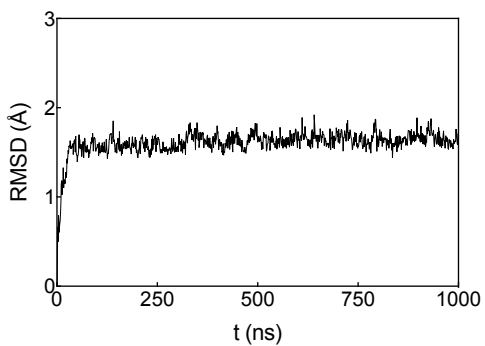

N487S

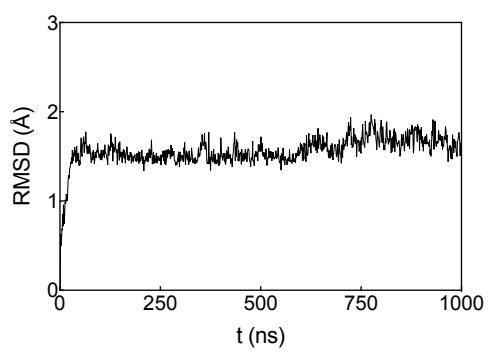

T500K

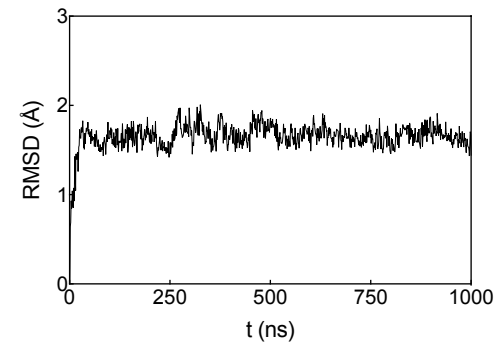

T500T

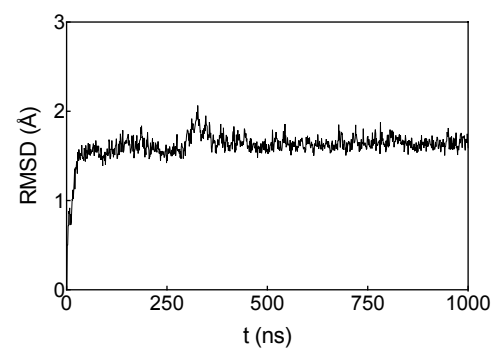

N487K

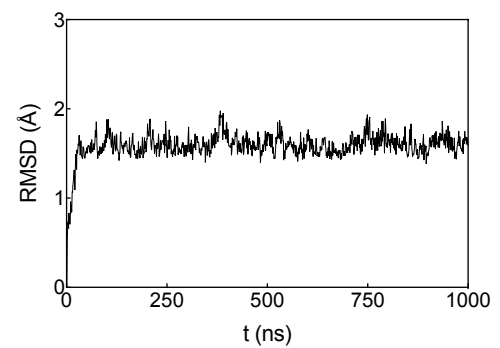

N487T

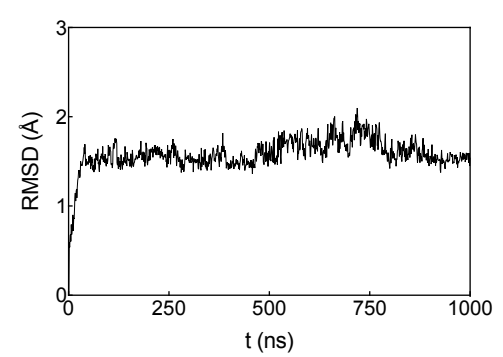

T500

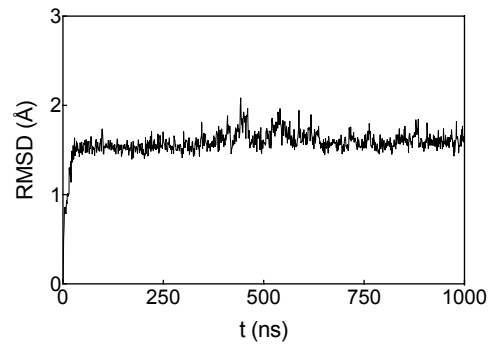

T500W

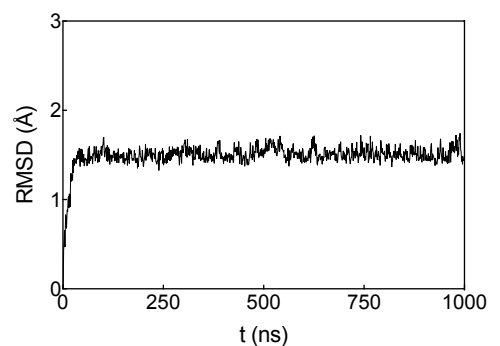

N487I

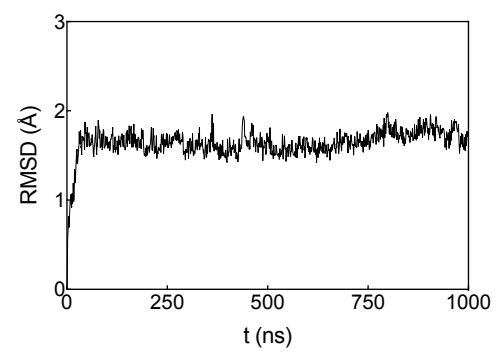

N487W

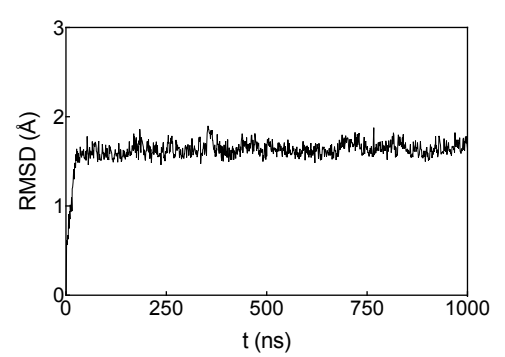


Y489D

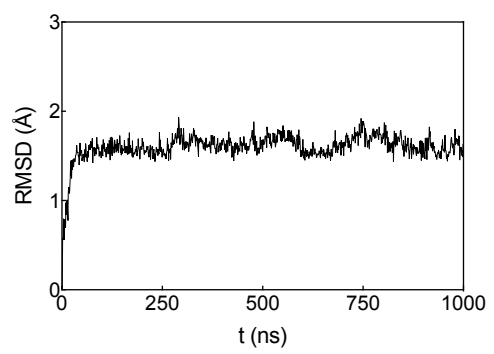

Y489S

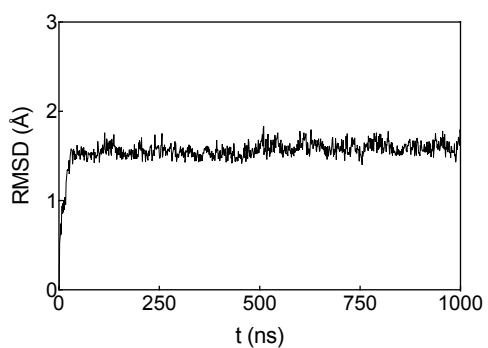

Y505D

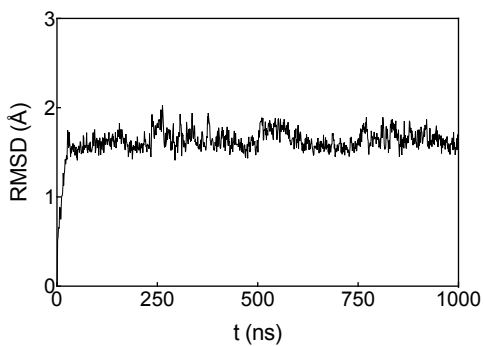

Y505S

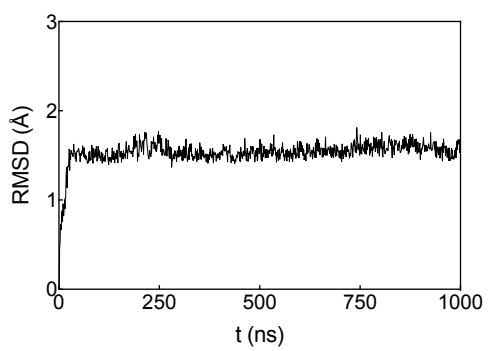

Y489K

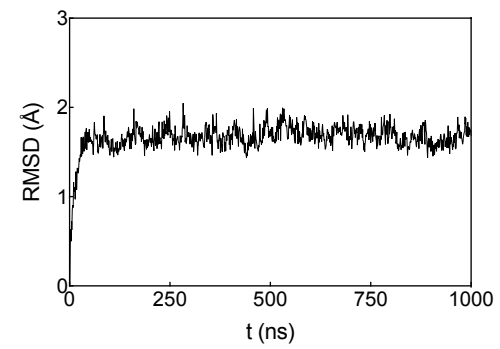

Y489T

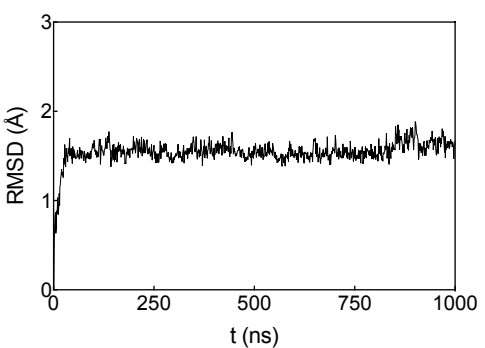

Y505K

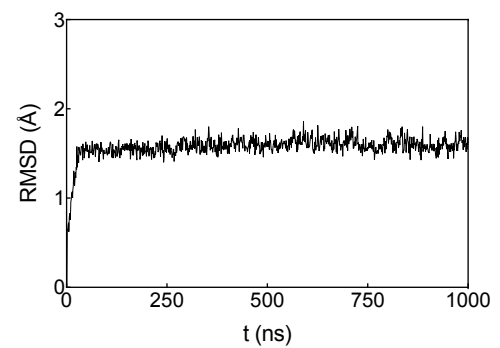

Y505T

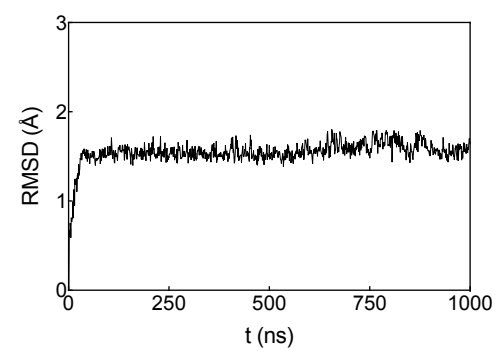

Y489I

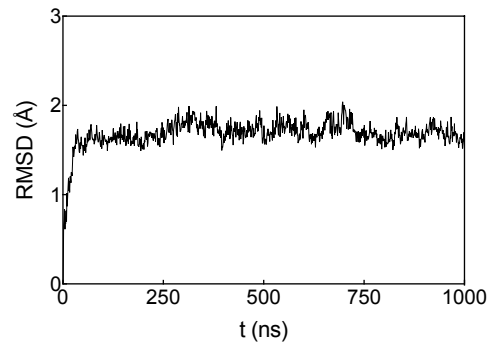

Y489W

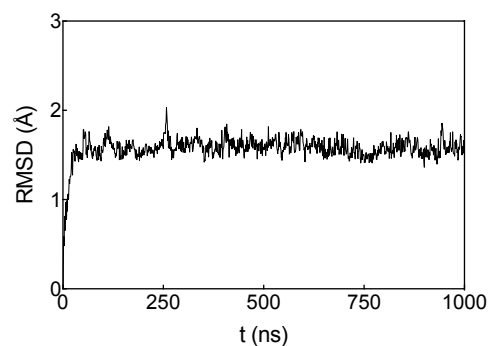

Y505I

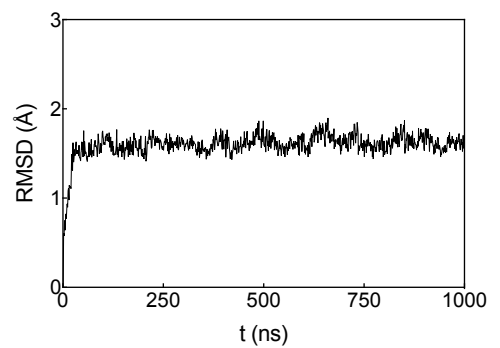

Y505W

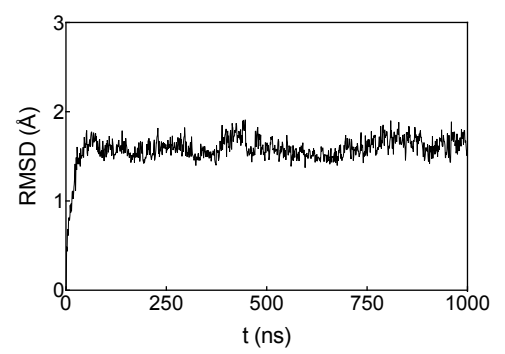


L455D

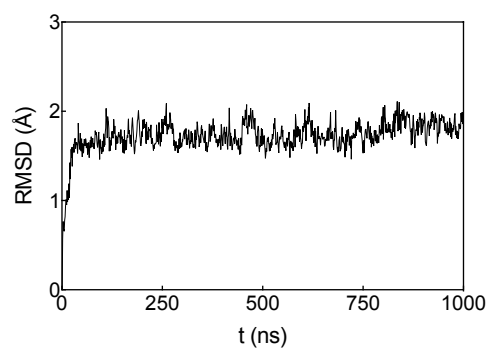

L455S

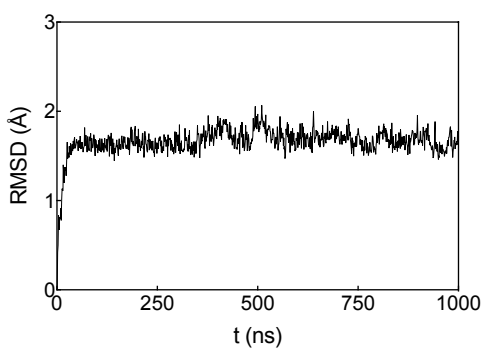

F456D

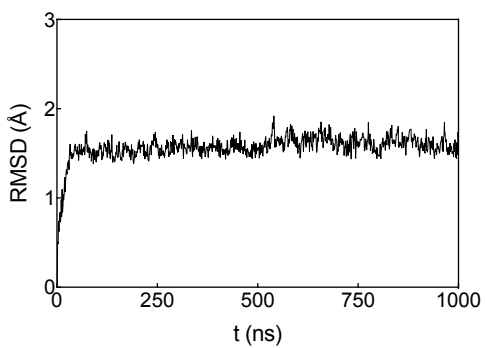

F456S

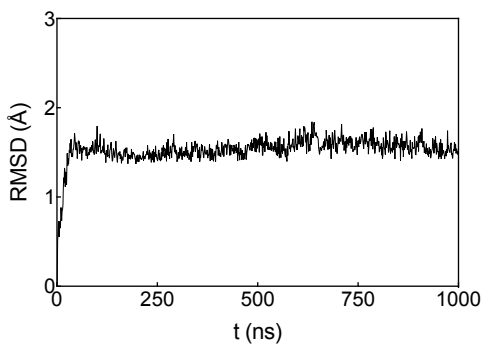

L455K

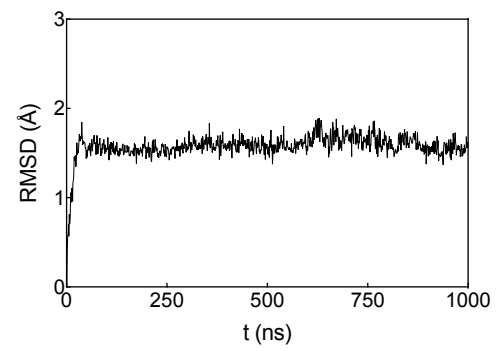

L455T

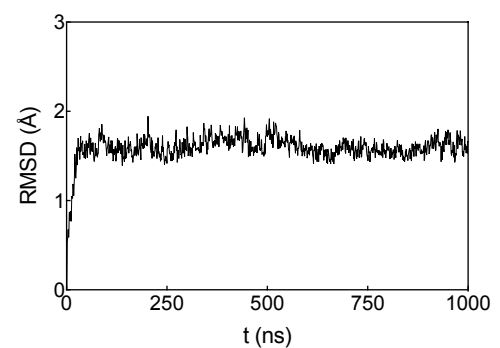

F456K

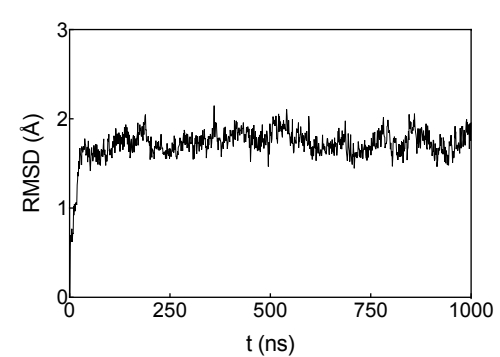

F456T

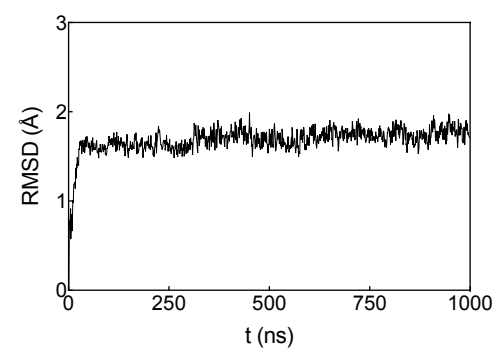

L455।

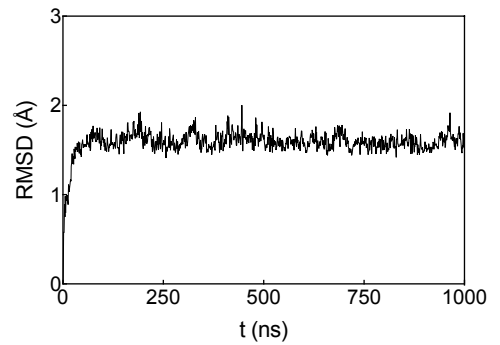

L455W

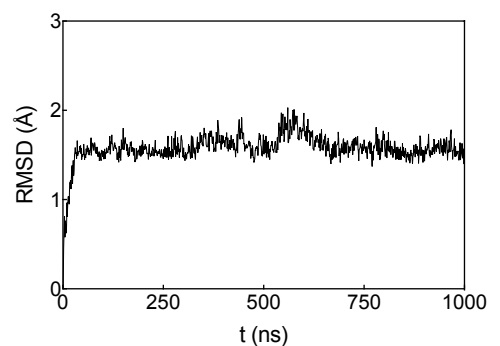

F456I

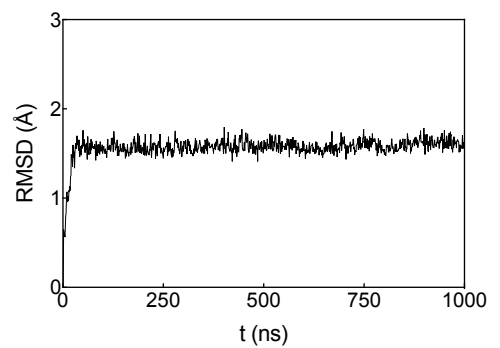

F456W

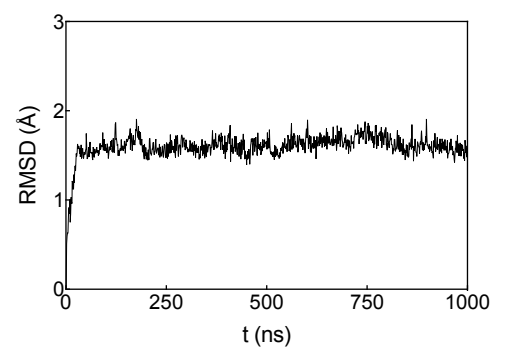


F486D

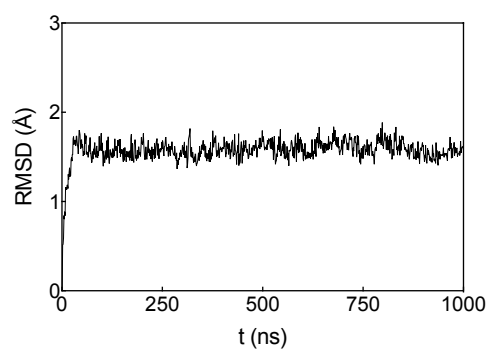

F486S

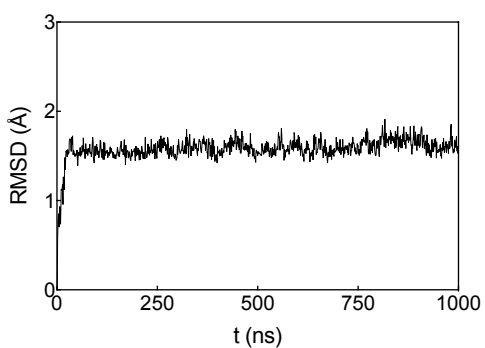

Q493D

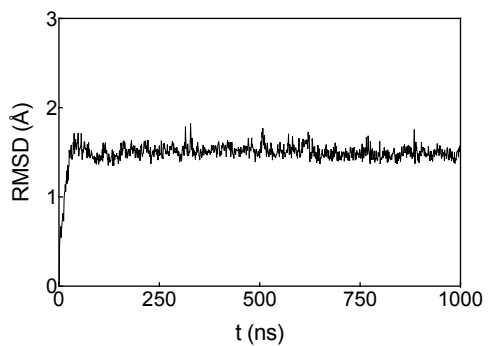

Q493S

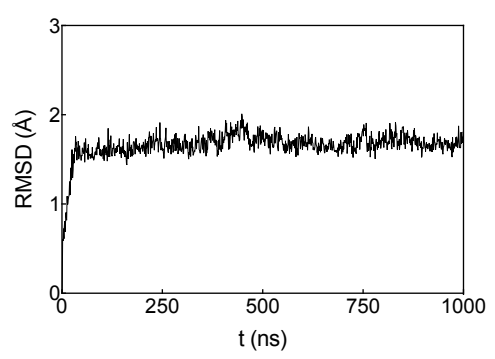

F486K

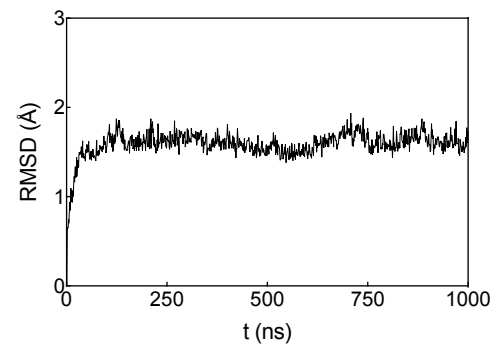

F486T

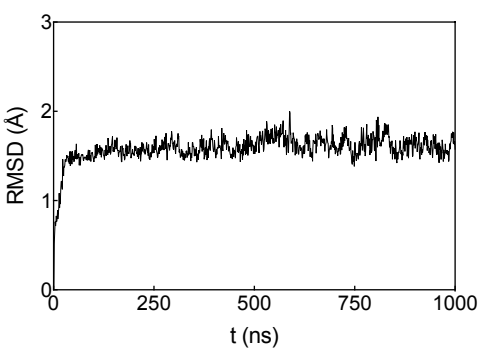

Q493K

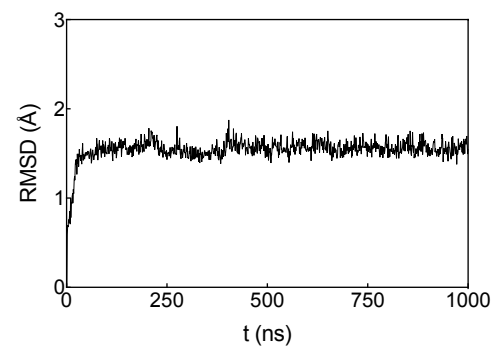

Q493T

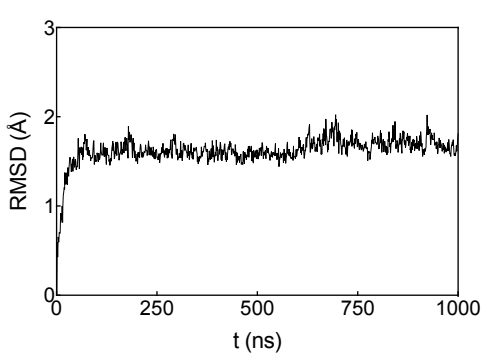

F486I

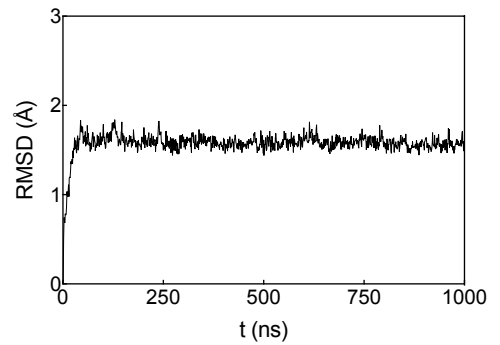

F486W

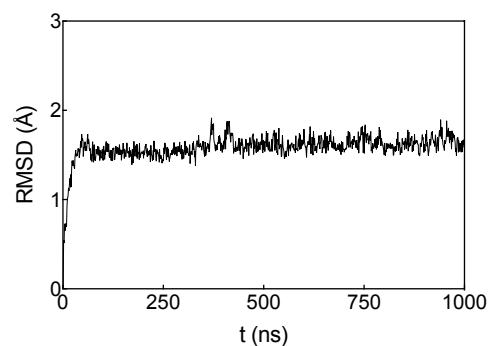

Q493।

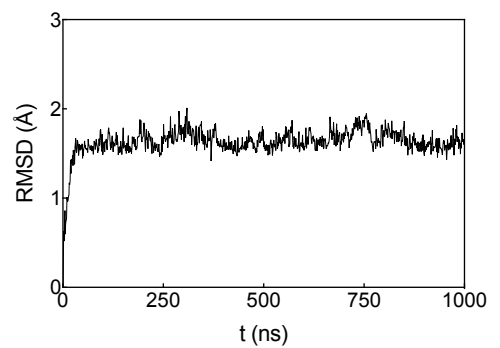

Q493W

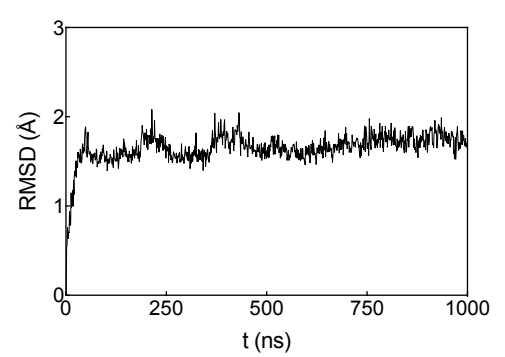


Q498D

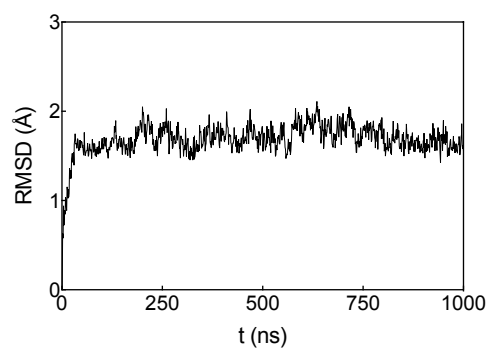

Q498S

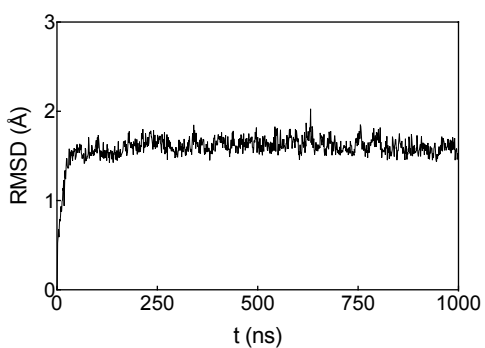

N501D

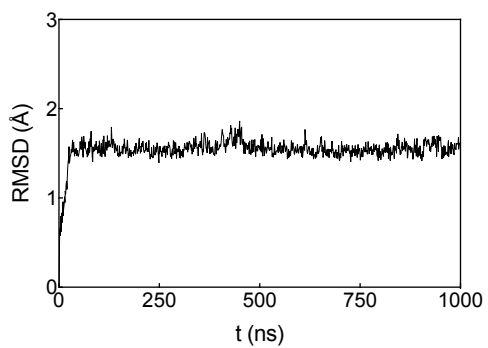

N501S

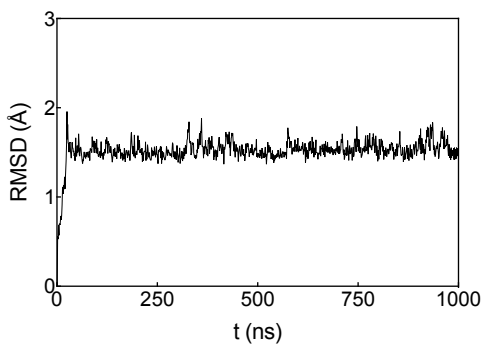

Q498K

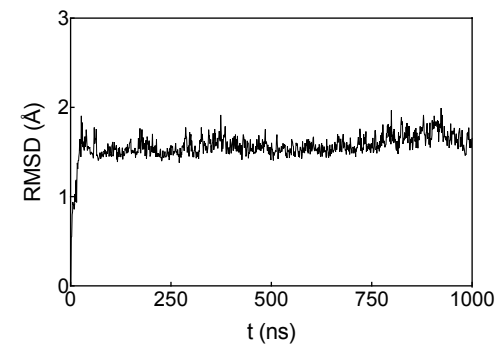

Q498T

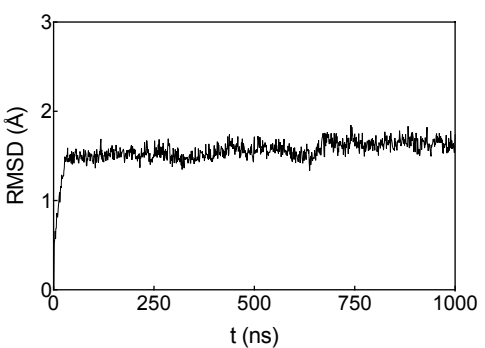

N501K

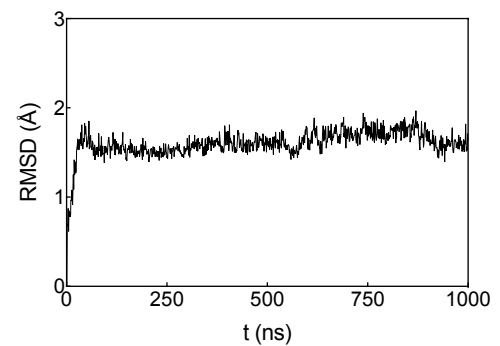

N501T

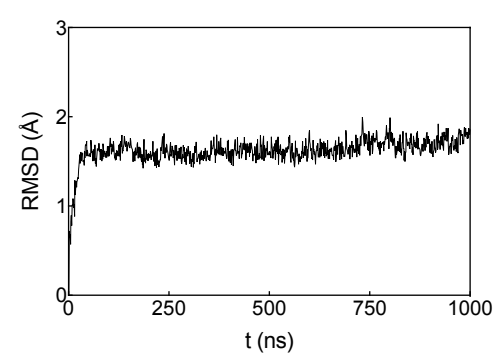

Q498|

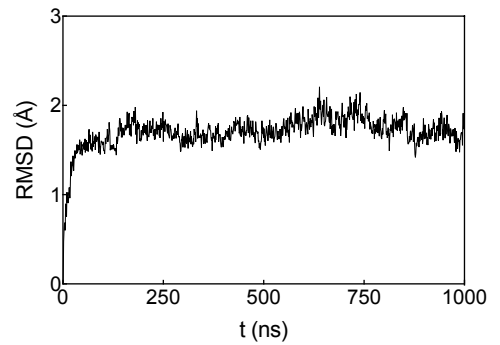

Q498W

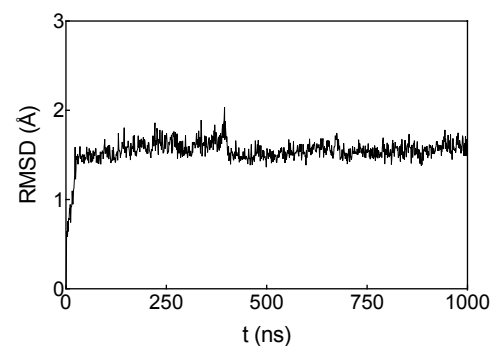

N501I

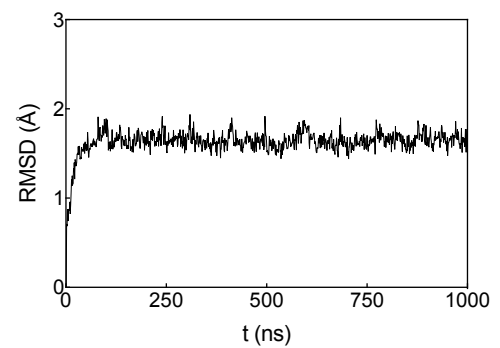

N501W

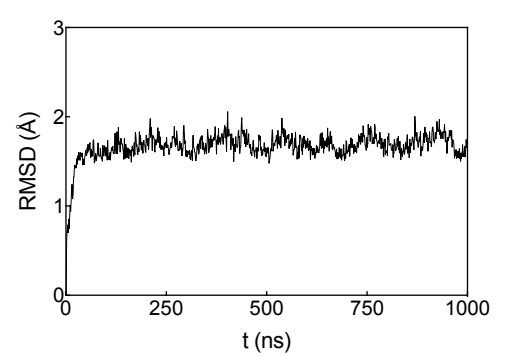


R403D

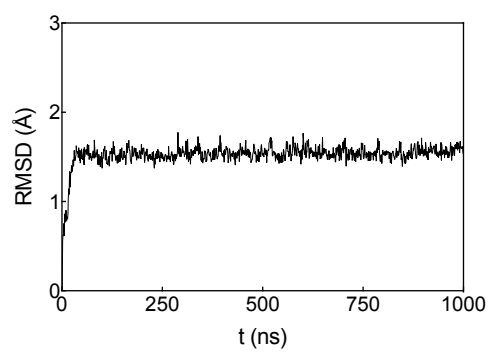

R403S

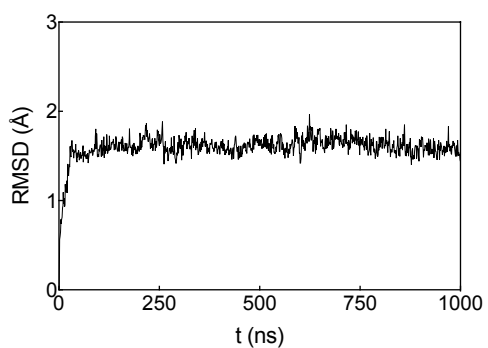

K417D

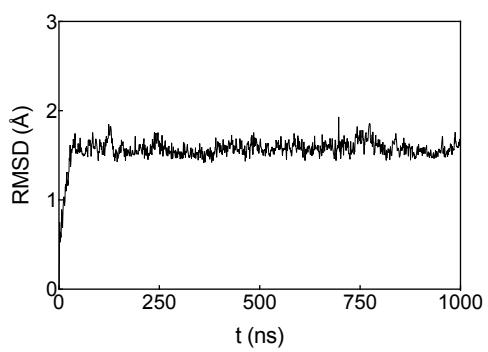

K417S

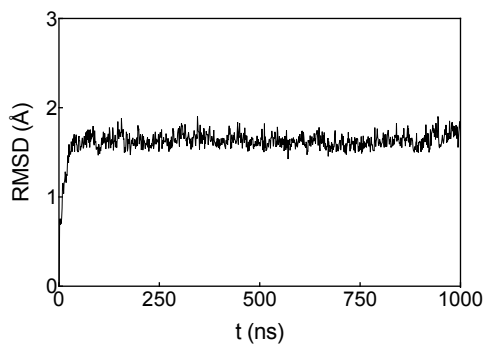

R403K

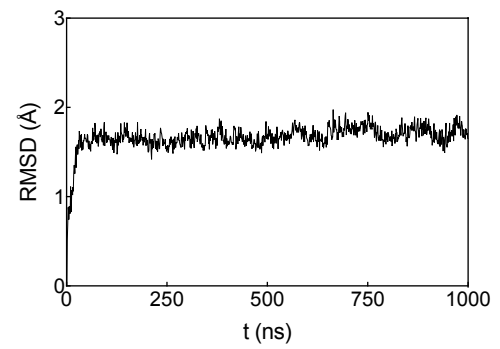

R403T

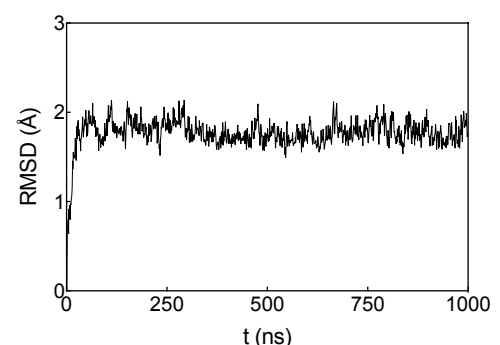

K417R

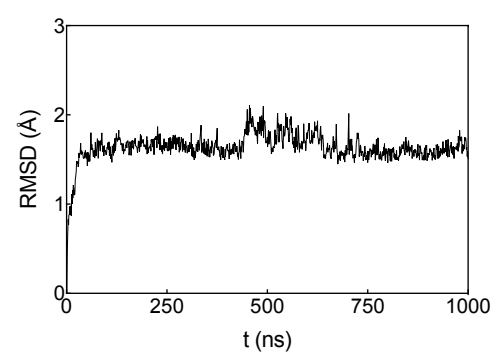

K417T

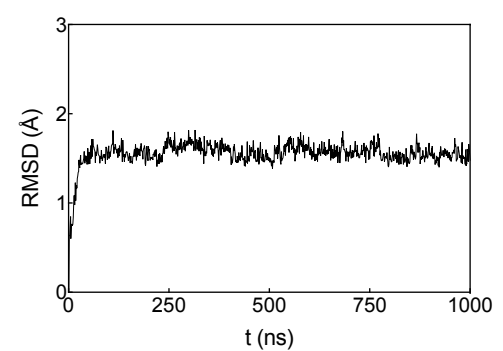

R403I

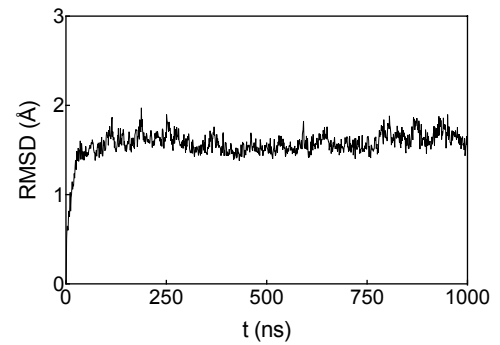

R403W

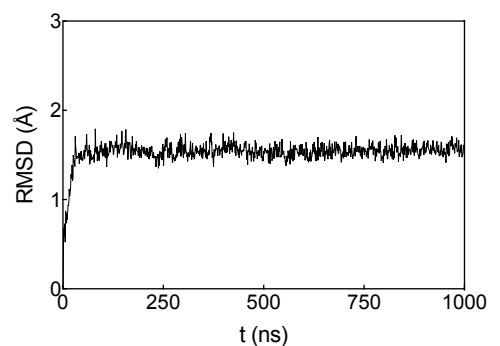

K417I

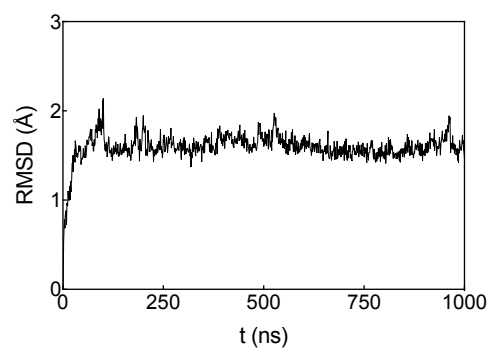

K417W

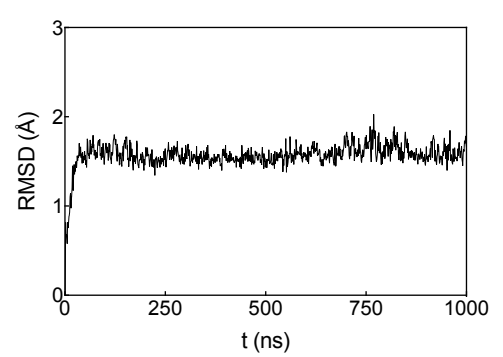

Figure S34. Root-mean-square deviation (RMSD) of $A C E 2 / S-R B D_{\text {cov-2 }}$ protein complex backbone atoms as a function of MD simulation time for all S-RBD $\mathrm{Cov}-2_{2}$ mutants. 


\section{Extended Methods Section}

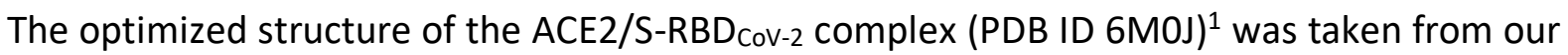
previous work. The coordinates files and force field parameters for the ACE $2 \mathrm{Zn}^{2+}$ binding site, and the input coordinate file (PDB) were already released as Supporting Information of our previous work $^{2}$ available at https://pubs.acs.org/doi/10.1021/acsnano.0c04674.

All calculations reported in this work were performed in AMBER19. ${ }^{3}$ The tleap software provided within AMBER19 was used to assign the $\mathrm{ff}^{14 \mathrm{SB}^{4}}$ and GLYCAM06j-1 ${ }^{5}$ forcefields to the starting protein/protein structure.

All ACE2 and S-RBD ${ }_{\text {Cov-2 }}$ mutations were introduced into the wild-type structure of the protein-protein complex by swapping the mutant residue into the specific site, according to a procedure we extensively validated through the years for different protein/protein and protein/ligand complexes. ${ }^{6-25}$

Each resultant protein/protein assembly was next subjected to solvation and equilibration protocols, as described in details in our previous work. Briefly, each complex was immersed in a box filled with TIP3PB ${ }^{26}$ water molecules and naturalized with the addition of a suitable number of $\mathrm{Na}^{+}$and $\mathrm{Cl}^{-}$counterions; next the ionic strength of the system was in order to mimic a physiological salt concentration $(0.15 \mathrm{M})$. Each supermolecular assembly was further optimized progressively removing initial restraints via a sequence of: 1) energy minimization (3000 steps of steepest descent followed by 3000 steps of conjugated gradient algorithms), 2) heating to $150 \mathrm{~K}$ (in $10 \mathrm{ps}$ of canonical ensemble (NVT) molecular dynamics (MD) followed by 50 ps MD simulation in the isothermal/isobaric ensemble (NPT, P = 1 atm, maintained by the Berendsen barostat ${ }^{27}$ ) to reach the target temperature of $300 \mathrm{~K}$; 3) further $10 \mathrm{~ns}$ in NPT conditions (phase 1) and 4) MD data production runs continued up to $1 \mu \mathrm{s}$, during which pressure was maintained using the Monte Carlo barostat implemented in AMBER (phase 2). Electrostatic interactions were computed by means of the particle mesh Ewald (PME) ${ }^{28}$ algorithm temperature was regulated by the Langevin method ${ }^{29}$ (collision frequency of $3 \mathrm{ps}^{-}$ $\left.{ }^{1}\right)$. The SHAKE algorithm ${ }^{30}$ was applied to allow a $2 \mathrm{fs}$ integration time step. All calculations were run with the pmemd module of AMBER19 running on the supercomputer Marconi100 (CINECA, Bologna, Italy) and on our CPU/GPU hybrid cluster. All images were produced by the UCSF Chimera software ${ }^{31}$ and on Prism 8 GraphPad Prism version 8.0.0 for Mac (GraphPad Software, San Diego, California USA, www.graphpad.com).

After the first $5 \mathrm{~ns}$ of the phase $2 \mathrm{MD}$ trajectory, $5 \mathrm{~ns}$ MD data were selected to calculate enthalpy and entropy contributions. Configurational sampling was preformed accordingly, with a time step of $10 \mathrm{fs}$; thus, a total of 500000 snapshots, sufficient for the interaction entropy (IE) calculations, ${ }^{32-34}$ were extracted from the relevant MD trajectory for the calculation of the protein/protein residue-specific interactions.

The free energy was calculated for each molecular species (protein/protein complex, ACE2, and S-RBD $\mathrm{COV}_{-2}$ ) in the framework of the MM/PBSA ansatz, ${ }^{35}$ and the protein/protein binding free energy was computed as the difference:

$\Delta G=G_{A C E 2 / S-R B D C O V-2}-\left(G_{A C E 2}+G_{S-R B D C O V-2}\right)=\Delta E_{v d W}+\Delta E_{E L E}+\Delta G_{S O L}-T \Delta S=\Delta H-T \Delta S$

in which $\Delta \mathrm{E}_{\mathrm{vdW}}$ and $\Delta \mathrm{E}_{\mathrm{ELE}}$ represent van der Waals and electrostatic molecular mechanics energies, and $\Delta G_{\text {sol }}$ includes the solvation free energy. The internal dielectric constant was set to the values of 2, 3 and 9 for nonpolar, polar, and charged residues, ${ }^{15,36}$ respectively. Lastly, the entropic contribution ( $T \Delta S$ ) was explicitly computed from the MD simulation by using the Interaction Entropy (IE) method. ${ }^{32-34}$ 
The role of the protein/protein interface key residues was calculated with the MM/PBSA method. Accordingly, the difference in the binding free energy between the wild-type (WT) protein and mutant counterpart, $\Delta \Delta \mathrm{G}$, is given by:

$\Delta \Delta \mathrm{G}=\Delta \mathrm{G}_{\text {WILD-TYPE }}-\Delta \mathrm{G}_{\text {MUTANT }}$

Thus, the adopted methodology allows for the estimation of the contribution of a given residue with respect to the overall protein-protein binding free energy; indeed, according to equation (5), a negative value of $\Delta \Delta \mathrm{G}$ indicated a favorable contribution for the wild type residue in that position and vice versa.

At the structural level, the stability of the main protein/protein interface intermolecular and intramolecular interactions detected during the MD simulation time interval adopted for the energetic analysis was assessed along the entire duration of the MD run.

\section{References}

1. Berman, H. M.; Westbrook, J.; Feng, Z.; Gilliland, G.; Bhat, T. N.; Weissig, H.;

Shindyalov, I. N.; Bourne, P. E., The Protein Data Bank. Nucleic Acids Res 2000, 28, 235-242.

2. Laurini, E.; Marson, D.; Aulic, S.; Fermeglia, M.; Pricl, S., Computational Alanine Scanning and Structural Analysis of the SARS-CoV-2 Spike Protein/Angiotensin-Converting Enzyme 2 Complex. ACS Nano 2020, 14, 11821-11830.

3. Case, D. A.; Ben-Shalom, I. Y.; Brozell, S. R.; Cerutti, D. S.; Cheatham, I., T.E. ; Cruzeiro, V. W. D.; Darden, T. A.; Duke, R. E.; Ghoreishi, D.; Giambasu, G.; Giese, T.; Gilson, M. K.; Gohlke, H.; Goetz, A. W.; Greene, D.; Harris, R.; Homeyer, N.; Huang, Y.; Izadi, S.; Kovalenko, A., et al. AMBER 2019, University of California, San Francisco, CA, USA. 2019.

4. Maier, J. A.; Martinez, C.; Kasavajhala, K.; Wickstrom, L.; Hauser, K. E.; Simmerling, C., ff14SB: Improving the Accuracy of Protein Side Chain and Backbone Parameters from ff99SB. J Chem Theory Comput 2015, 11, 3696-3713.

5. Kirschner, K. N.; Yongye, A. B.; Tschampel, S. M.; Gonzalez-Outeirino, J.; Daniels, C. R.; Foley, B. L.; Woods, R. J., GLYCAM06: A Generalizable Biomolecular Force Field. Carbohydrates. J Comput Chem 2008, 29, 622-655.

6. Pricl, S.; Fermeglia, M.; Ferrone, M.; Tamborini, E., T315I-Mutated Bcr-Abl in Chronic Myeloid Leukemia and Imatinib: Insights from a Computational Study. Mol Cancer Ther 2005, 4, 1167-1174.

7. $\quad$ Ferrone, M.; Perrone, F.; Tamborini, E.; Paneni, M. S.; Fermeglia, M.; Suardi, S.; Pastore, E.; Delia, D.; Pierotti, M. A.; Pricl, S.; Pilotti, S., Functional Analysis and Molecular Modeling Show a Preserved Wild-Type Activity of p53(C238Y). Mol Cancer Ther 2006, 5, 1467-1473.

8. Tamborini, E.; Pricl, S.; Negri, T.; Lagonigro, M. S.; Miselli, F.; Greco, A.; Gronchi, A.; Casali, P. G.; Ferrone, M.; Fermeglia, M.; Carbone, A.; Pierotti, M. A.; Pilotti, S., Functional Analyses and Molecular Modeling of Two C-Kit Mutations Responsible for Imatinib Secondary Resistance in GIST Patients. Oncogene 2006, 25, 6140-6146.

9. McAuliffe, J. C.; Wang, W. L.; Pavan, G. M.; Pricl, S.; Yang, D.; Chen, S. S.; Lazar, A. J.; Pollock, R. E.; Trent, J. C., Unlucky Number 13? Differential Effects of KIT Exon 13 Mutation in Gastrointestinal Stromal Tumors. Mol Oncol 2008, 2, 161-163.

10. Conca, E.; Negri, T.; Gronchi, A.; Fumagalli, E.; Tamborini, E.; Pavan, G. M.; Fermeglia, M.; Pierotti, M. A.; Pricl, S.; Pilotti, S., Activate and Resist: L576P-KIT in GIST. Mol Cancer Ther 2009, 8, 2491-2495. 
11. Negri, T.; Pavan, G. M.; Virdis, E.; Greco, A.; Fermeglia, M.; Sandri, M.; Pricl, S.; Pierotti, M. A.; Pilotti, S.; Tamborini, E., T670X KIT Mutations in Gastrointestinal Stromal Tumors: Making Sense of Missense. J Natl Cancer Inst 2009, 101, 194-204.

12. Woodman, S. E.; Trent, J. C.; Stemke-Hale, K.; Lazar, A. J.; Pricl, S.; Pavan, G. M.; Fermeglia, M.; Gopal, Y. N.; Yang, D.; Podoloff, D. A.; Ivan, D.; Kim, K. B.; Papadopoulos, N.; Hwu, P.; Mills, G. B.; Davies, M. A., Activity of Dasatinib against L576P KIT Mutant Melanoma: Molecular, Cellular, and Clinical Correlates. Mol Cancer Ther 2009, 8, 2079-2085. 13. Dileo, P.; Pricl, S.; Tamborini, E.; Negri, T.; Stacchiotti, S.; Gronchi, A.; Posocco, P.; Laurini, E.; Coco, P.; Fumagalli, E.; Casali, P. G.; Pilotti, S., Imatinib Response in Two GIST Patients Carrying Two Hitherto Functionally Uncharacterized PDGFRA Mutations: An Imaging, Biochemical and Molecular Modeling Study. Int J Cancer 2011, 128, 983-990. 14. Bozzi, F.; Conca, E.; Laurini, E.; Posocco, P.; Lo Sardo, A.; Jocollè, G.; Sanfilippo, R.; Gronchi, A.; Perrone, F.; Tamborini, E.; Pelosi, G.; Pierotti, M. A.; Maestro, R.; Pricl, S.; Pilotti, S., In Vitro and in Silico Studies of MDM2/MDMX Isoforms Predict Nutlin-3A Sensitivity in Well/De-Differentiated Liposarcomas. Lab Invest 2013, 93, 1232-1240.

15. Conca, E.; Miranda, C.; Dal Col, V.; Fumagalli, E.; Pelosi, G.; Mazzoni, M.; Fermeglia, M.; Laurini, E.; Pierotti, M. A.; Pilotti, S.; Greco, A.; Pricl, S.; Tamborini, E., Are Two Better than One? A Novel Double-Mutant KIT in GIST that Responds to Imatinib. Mol Oncol 2013, 7, 756-762.

16. Laurini, E.; Posocco, P.; Fermeglia, M.; Gibbons, D. L.; Quintás-Cardama, A.; Pricl, S., Through the Open Door: Preferential Binding of Dasatinib to the Active Form of BCR-ABL Unveiled by in Silico Experiments. Mol Oncol 2013, 7, 968-975.

17. Brune, S.; Schepmann, D.; Klempnauer, K. H.; Marson, D.; Dal Col, V.; Laurini, E.; Fermeglia, M.; Wünsch, B.; Pricl, S., The Sigma Enigma: In Vitro/in Silico Site-Directed Mutagenesis Studies Unveil o1 Rceptor Lgand Binding. Biochemistry 2014, 53, 2993-3003. 18. Gibbons, D. L.; Pricl, S.; Posocco, P.; Laurini, E.; Fermeglia, M.; Sun, H.; Talpaz, M.; Donato, N.; Quintás-Cardama, A., Molecular Dynamics Reveal BCR-ABL1 Polymutants as a Unique Mechanism of Resistance to PAN-BCR-ABL1 Kinase Inhibitor Therapy. Proc Natl Acad Sci U S A 2014, 111, 3550-3555.

19. Brambilla, L.; Genini, D.; Laurini, E.; Merulla, J.; Perez, L.; Fermeglia, M.; Carbone, G. M.; Pricl, S.; Catapano, C. V., Hitting the Rght Spot: Mechanism of Action of OPB-31121, a Novel and Potent Inhibitor of the Signal Transducer and Activator of Transcription 3 (STAT3). Mol Oncol 2015, 9, 1194-1206.

20. Morgan, A.; Gandin, I.; Belcaro, C.; Palumbo, P.; Palumbo, O.; Biamino, E.; Dal Col, V.; Laurini, E.; Pricl, S.; Bosco, P.; Carella, M.; Ferrero, G. B.; Romano, C.; d'Adamo, A. P.; Faletra, F.; Vozzi, D., Target Sequencing Approach Intended to Discover New Mutations in NonSyndromic Intellectual Disability. Mutat Res 2015, 781, 32-36.

21. Pricl, S.; Cortelazzi, B.; Dal Col, V.; Marson, D.; Laurini, E.; Fermeglia, M.; Licitra, L.; Pilotti, S.; Bossi, P.; Perrone, F., Smoothened (SMO) Receptor Mutations Dictate Resistance to Vismodegib in Basal Cell Carcinoma. Mol Oncol 2015, 9 (2), 389-397.

22. Colombo, C.; Belfiore, A.; Paielli, N.; De Cecco, L.; Canevari, S.; Laurini, E.; Fermeglia, M.; Pricl, S.; Verderio, P.; Bottelli, S.; Fiore, M.; Stacchiotti, S.; Palassini, E.; Gronchi, A.; Pilotti, S.; Perrone, F., $\beta$-Catenin in Desmoid-Type Fibromatosis: Deep Insights into the Role of T41A and S45F Mutations on Protein Structure and Gene Expression. Mol Oncol 2017, 11, 1495-1507.

23. Genini, D.; Brambilla, L.; Laurini, E.; Merulla, J.; Civenni, G.; Pandit, S.; D'Antuono, R.; Perez, L.; Levy, D. E.; Pricl, S.; Carbone, G. M.; Catapano, C. V., Mitochondrial Dysfunction 
Induced by a SH2 Domain-Targeting STAT3 Inhibitor Leads to Metabolic Synthetic Lethality in Cancer Cells. Proc Natl Acad Sci U S A 2017, 114, e4924-e4933.

24. Perfetti, V.; Laurini, E.; Aulić, S.; Fermeglia, M.; Riboni, R.; Lucioni, M.; Dallera, E.; Delfanti, S.; Pugliese, L.; Latteri, F. S.; Pietrabissa, A.; Pricl, S., Molecular and Functional Characterization of a New 3' End KIT Juxtamembrane Deletion in a Duodenal GIST Treated with Neoadjuvant Imatinib. Oncotarget 2017, 8, 56158-56167.

25. Ziouziou, H.; Andrieu, C.; Laurini, E.; Karaki, S.; Fermeglia, M.; Oueslati, R.; Taieb, D.; Camplo, M.; Siri, O.; Pricl, S.; Katsogiannou, M.; Rocchi, P., Targeting Hsp27/elF4E Interaction with Phenazine Compound: A Promising Alternative for Castration-Resistant Prostate Cancer Treatment. Oncotarget 2017, 8, 77317-77329.

26. Jorgensen, W. L.; Chandrasekhar, J.; Madura, J. D.; Impey, R. W.; Klein, M. L., Comparison of Simple Potential Functions for Simulating Liquid Water. J Chem Phys 1983, 79 (2), 926-935.

27. Berendsen, H. J. C.; Postma, J. P. M.; van Gunsteren, W. F.; DiNola, A.; Haak, J. R., Molecular Dynamics with Coupling to an External Bath. J Chem Phys 1984, 81, 3684-3690.

28. Toukmaji, A.; Sagui, C.; Board, J.; Darden, T., Efficient Particle-Mesh Ewald Based Approach to Fixed and Induced Dipolar Interactions. J Chem Phys 2000, 113, 10913-10927.

29. Loncharich, R. J.; Brooks, B. R.; Pastor, R. W., Langevin Dynamics of Peptides: The Frictional Dependence of Isomerization Rates of N-Acetylalanyl-N'-Methylamide.

Biopolymers 1992, 32, 523-535.

30. Ryckaert, J.-P.; Ciccotti, G.; Berendsen, H. J. C., Numerical Integration of the Cartesian Equations of Motion of a System with Constraints: Molecular Dynamics of NAlkanes. J Comput Phys 1977, 23, 327-341.

31. Pettersen, E. F.; Goddard, T. D.; Huang, C. C.; Couch, G. S.; Greenblatt, D. M.; Meng, E. C.; Ferrin, T. E., UCSF Chimera--A Visualization System for Exploratory Research and Analysis. J Comput Chem 2004, 25, 1605-1612.

32. Yan, Y.; Yang, M.; Ji, C. G.; Zhang, J. Z. H., Interaction Entropy for Computational Alanine Scanning. J Chem Inf Model 2017, 57, 1112-1122.

33. Sun, Z.; Yan, Y. N.; Yang, M.; Zhang, J. Z., Interaction Entropy for Protein-Protein Binding. J Chem Phys 2017, 146, 124124.

34. Liu, X.; Peng, L.; Zhou, Y.; Zhang, Y.; Zhang, J. Z. H., Computational Alanine Scanning with Interaction Entropy for Protein-Ligand Binding Free Energies. $J$ Chem Theory Comput 2018, 14, 1772-1780.

35. Wang, E.; Sun, H.; Wang, J.; Wang, Z.; Liu, H.; Zhang, J. Z. H.; Hou, T., End-Point Binding Free Energy Calculation with MM/PBSA and MM/GBSA: Strategies and Applications in Drug Design. Chem Rev 2019, 119, 9478-9508.

36. Yan, Y.; Yang, M.; Ji, C. G.; Zhang, J. Z. H., Interaction Entropy for Computational Alanine Scanning. J Chem Inf Model 2017, 57, 1112-1122. 